\title{
THE MINKOWSKI QUESTION MARK FUNCTION: EXPLICIT SERIES FOR THE DYADIC PERIOD FUNCTION AND MOMENTS
}

\author{
GIEDRIUS ALKAUSKAS
}

\begin{abstract}
Previously, several natural integral transforms of the Minkowski question mark function $F(x)$ were introduced by the author. Each of them is uniquely characterized by certain regularity conditions and the functional equation, thus encoding intrinsic information about $F(x)$. One of them, the dyadic period function $G(z)$, was defined as a Stieltjes transform. In this paper we introduce a family of "distributions" $F_{\mathrm{p}}(x)$ for $\Re \mathrm{p} \geq 1$, such that $F_{1}(x)$ is the question mark function and $F_{2}(x)$ is a discrete distribution with support on $x=1$. We prove that the generating function of moments of $F_{\mathrm{p}}(x)$ satisfies the three-term functional equation. This has an independent interest, though our main concern is the information it provides about $F(x)$. This approach yields the following main result: we prove that the dyadic period function is a sum of infinite series of rational functions with rational coefficients.
\end{abstract}

\section{INTRODUCTION AND MAIN RESULT}

The aim of this paper is to continue investigations on the moments of the Minkowski ?(x) function, begun in [1, [2] and [3]. The function ? $(x)$ ("the question mark function") was introduced by Minkowski as an example of a continuous function $F:[0, \infty) \rightarrow[0,1)$, which maps rationals to dyadic rationals, and quadratic irrationals to nondyadic rationals. For a nonnegative real $x$ it is defined by the expression

$$
F\left(\left[a_{0}, a_{1}, a_{2}, a_{3}, \ldots\right]\right)=1-2^{-a_{0}}+2^{-\left(a_{0}+a_{1}\right)}-2^{-\left(a_{0}+a_{1}+a_{2}\right)}+\ldots,
$$

where $x=\left[a_{0}, a_{1}, a_{2}, a_{3}, \ldots\right]$ stands for the representation of $x$ by a (regular) continued fraction [15]. By tradition, this function is more often investigated in the interval $[0,1]$. Accordingly, we make a convention that $?(x)=2 F(x)$ for $x \in[0,1]$. For rational $x$, the series terminates at the last nonzero partial quotient $a_{n}$ of the continued fraction. This function is continuous, monotone and singular [9]. A by far not complete overview of the papers written about the Minkowski question mark function or closely related topics (Farey tree, enumeration of rationals, Stern's diatomic sequence, various 1-dimensional generalizations and generalizations to higher dimensions, statistics of denominators and Farey intervals, Hausdorff dimension and analytic properties) can be found in [1. These works include

Received by the editor September 15, 2008 and, in revised form, January 17, 2009.

2000 Mathematics Subject Classification. Primary 11A55, 26A30, 32A05; Secondary 40A15, 37F50, $11 \mathrm{~F} 37$.

Key words and phrases. The Minkowski question mark function, the dyadic period function, three-term functional equation, analytic theory of continued fractions, Julia sets, the Farey tree. 
[5], 6], 8], 9], 10, 12, 13] (this is the only paper where the moments of a certain singular distribution, a close relative of $F(x)$, were considered), 11, [14, 16], 18], [20, [24, 25], 26], 27, 28, 29], 30, 31, 33. The internet page 36] contains an up-to-date and exhaustive bibliographical list of papers related to the Minkowski question mark function.

Recently, Calkin and Wilf 8 defined a binary tree which is generated by the iteration

$$
\frac{a}{b} \mapsto \frac{a}{a+b}, \frac{a+b}{b},
$$

starting from the root $\frac{1}{1}$. The last two authors have greatly publicized this tree, but it was known long ago to physicists and mathematicians (alias, Stern-Brocot or Farey tree). Elementary considerations show that this tree contains every positive rational number once and only once, each being represented in lowest terms. The first four iterations lead to

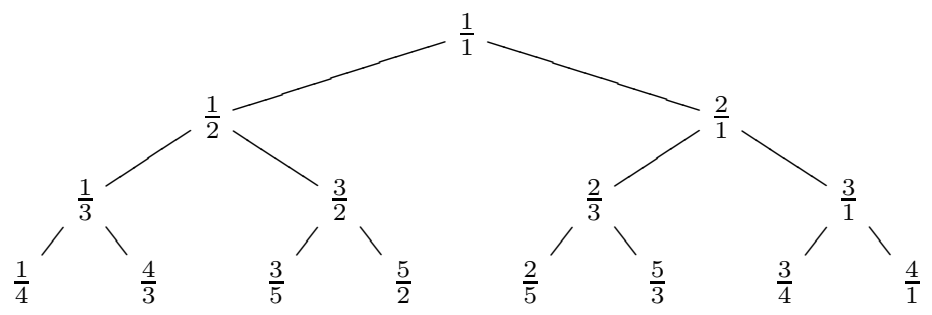

It is of utmost importance to note that the $n$th generation consists of exactly those $2^{n-1}$ positive rational numbers, whose elements of the continued fraction sum up to $n$. This fact can be easily inherited directly from the definition. First, if the rational number $\frac{a}{b}$ is represented as a continued fraction $\left[a_{0}, a_{1}, \ldots, a_{r}\right]$, then the map $\frac{a}{b} \rightarrow \frac{a+b}{b}$ maps $\frac{a}{b}$ to $\left[a_{0}+1, a_{1} \ldots, a_{r}\right]$. Second, the map $\frac{a}{b} \rightarrow \frac{a}{a+b}$ maps $\frac{a}{b}$ to $\left[0, a_{1}+1, \ldots, a_{r}\right]$ in case $\frac{a}{b}<1$, and to $\left[0,1, a_{0}, a_{1}, \ldots, a_{r}\right]$ in case $\frac{a}{b}>1$. This is an important fact which makes the investigations of rational numbers according to their position in the Calkin-Wilf tree highly motivated from the perspective of metric number theory and the dynamics of continued fractions.

It is well known that each generation of (2) possesses a distribution function $F_{n}(x)$, and $F_{n}(x)$ converges uniformly to $F(x)$. The function $F(x)$ as a distribution function (in the sense of probability theory, which imposes the condition of monotonicity) is uniquely determined by the functional equation [1]

$$
2 F(x)=\left\{\begin{array}{cl}
F(x-1)+1 & \text { if } \quad x \geq 1 \\
F\left(\frac{x}{1-x}\right) & \text { if } \quad 0 \leq x<1 .
\end{array}\right.
$$

This implies $F(x)+F(1 / x)=1$. The mean value of $F(x)$ has been investigated by several authors, and was proved to be $3 / 2$.

Lastly, and most importantly, let us point out that, surprisingly, there are striking similarities and parallels between the results proved in [1] and 2] with LewisZagier's (22, 23]) results on period functions for Maass wave forms. (See 22 for the explanation of this phenomena.) 
Just before formulating the main theorem of this paper, we provide a short summary of previous results proved by the author about certain natural integral transforms of $F(x)$. Let

$$
M_{L}=\int_{0}^{\infty} x^{L} \mathrm{~d} F(x), \quad m_{L}=\int_{0}^{\infty}\left(\frac{x}{x+1}\right)^{L} \mathrm{~d} F(x)=2 \int_{0}^{1} x^{L} \mathrm{~d} F(x) .
$$

Both sequences are of definite number-theoretical significance because

$$
M_{L}=\lim _{n \rightarrow \infty} 2^{1-n} \sum_{a_{0}+a_{1}+\ldots+a_{s}=n}\left[a_{0}, a_{1}, . ., a_{s}\right]^{L}, \quad m_{L}=\lim _{n \rightarrow \infty} 2^{2-n} \sum_{a_{1}+\ldots+a_{s}=n}\left[0, a_{1}, . ., a_{s}\right]^{L},
$$

(the summation takes place over rational numbers represented as continued fractions; thus, $a_{0} \geq 0, a_{i} \geq 1$ and $a_{s} \geq 2$ ). We define the exponential generating functions

$$
\begin{aligned}
M(t) & =\sum_{L=0}^{\infty} \frac{M_{L}}{L !} t^{L}=\int_{0}^{\infty} e^{x t} \mathrm{~d} F(x), \\
\mathfrak{m}(t) & =\sum_{L=0}^{\infty} \frac{m_{L}}{L !} t^{L}=\int_{0}^{\infty} \exp \left(\frac{x t}{x+1}\right) \mathrm{d} F(x)=2 \int_{0}^{1} e^{x t} \mathrm{~d} F(x) .
\end{aligned}
$$

One directly verifies that $\mathfrak{m}(t)$ is an entire function, and that $M(t)$ is a meromorphic function with simple poles at $z=\log 2+2 \pi i n, n \in \mathbb{Z}$. Further, we have

$$
M(t)=\frac{\mathfrak{m}(t)}{2-e^{t}}, \quad \mathfrak{m}(t)=e^{t} \mathfrak{m}(-t) .
$$

The second identity represents only the symmetry property, given by $F(x)+F(1 / x)$ $=1$. The main result about $\mathfrak{m}(t)$ is that it is uniquely determined by the regularity condition $\mathfrak{m}(-t) \ll e^{-\sqrt{\log 2} \sqrt{t}}$, as $t \rightarrow \infty$, the boundary condition $\mathfrak{m}(0)=1$, and the integral equation

$$
\mathfrak{m}(-s)=\left(2 e^{s}-1\right) \int_{0}^{\infty} \mathfrak{m}^{\prime}(-t) J_{0}(2 \sqrt{s t}) \mathrm{d} t, \quad s \in \mathbb{R}_{+} .
$$

(Here $J_{0}(\star)$ stands for the Bessel function $J_{0}(z)=\frac{1}{\pi} \int_{0}^{\pi} \cos (z \sin x) \mathrm{d} x$.)

Our primary object of investigation is the generating function of moments. Let $G(z)=\sum_{L=1}^{\infty} m_{L} z^{L-1}$. This series converges for $|z| \leq 1$, and the functional equation for $G(z)$ (see below) implies that all the derivatives of $G(z)$ exist at $z=1$ if we approach this point while remaining in the domain $\Re z \leq 1$. Then the integral

$$
G(z)=\int_{0}^{\infty} \frac{1}{x+1-z} \mathrm{~d} F(x)=2 \int_{0}^{1} \frac{x}{1-x z} \mathrm{~d} F(x)
$$

(which is a Stieltjes transform of $F(x)$ ) extends $G(z)$ to the cut plane $\mathbb{C} \backslash(1, \infty)$. The generating function of moments $M_{L}$ does not exist due to the factorial growth of $M_{L}$, but this generating function can still be defined in the cut plane $\mathbb{C}^{\prime}=\mathbb{C} \backslash(0, \infty)$ 
by $\int_{0}^{\infty} \frac{x}{1-x z} \mathrm{~d} F(x)$. In fact, this integral just equals $G(z+1)$. Thus, there exist all higher derivatives of $G(z)$ at $z=1$, and $\left.\frac{1}{(L-1) !} \frac{\mathrm{d}^{L-1}}{\mathrm{~d} z^{L-1}} G(z)\right|_{z=1}=M_{L}, L \geq 1$. The following result was proved in [1].

Theorem 1.1. The function $G(z)$, defined initially as a power series, has an analytic continuation to the cut plane $\mathbb{C} \backslash(1, \infty)$ via (5). It satisfies the functional equation

$$
\frac{1}{z}+\frac{1}{z^{2}} G\left(\frac{1}{z}\right)+2 G(z+1)=G(z)
$$

and also the symmetry property

$$
G(z+1)=-\frac{1}{z^{2}} G\left(\frac{1}{z}+1\right)-\frac{1}{z}
$$

(which is a consequence of the main functional equation). Moreover, $G(z) \rightarrow 0$ if $z \rightarrow \infty$ and the distance from $z$ to a half-line $[0, \infty)$ tends to infinity. Conversely, the function having these properties is unique.

Accordingly, this result and the specific appearance of the three-term functional equation justifies the name for $G(z)$ as the dyadic period function.

We wish to emphasize that the main motivation for previous research was clarification of the nature and structure of the moments $m_{L}$. It was greatly desirable to give these constants (emerging as if from geometric chaos) some expression other than the one obtained directly from the Farey (or Calkin-Wilf) tree, which could reveal their structure to a greater extent. This is accomplished in the current work. Thus, the main result can be formulated as follows.

Theorem 1.2. There exist canonical and explicit sequence of rational functions $\mathbf{H}_{n}(z)$, such that for $\left\{|z| \leq \frac{3}{4}\right\} \cup\left\{\left|z+\frac{9}{7}\right| \leq \frac{12}{7}\right\}$ one has an absolutely convergent series

$$
G(z)=\int_{0}^{\infty} \frac{1}{x+1-z} \mathrm{~d} F(x)=\sum_{n=0}^{\infty}(-1)^{n} \mathbf{H}_{n}(z), \quad \mathbf{H}_{n}(z)=\frac{\mathscr{B}_{n}(z)}{(z-2)^{n+1}}
$$

where $\mathscr{B}_{n}(z)$ is polynomial with rational coefficients of degree $n-1$. For $n \geq 1$ it has the following reciprocity property:

$$
\mathscr{B}_{n}(z+1)=(-1)^{n} z^{n-1} \mathscr{B}_{n}\left(\frac{1}{z}+1\right), \quad \mathscr{B}_{n}(0)=0 .
$$

The rational functions $\mathbf{H}_{n}(z)$ are defined via implicit and rather complicated recurrence (27) (see Section 6). The following table gives the initial polynomials $\mathscr{B}_{n}(z)$. 


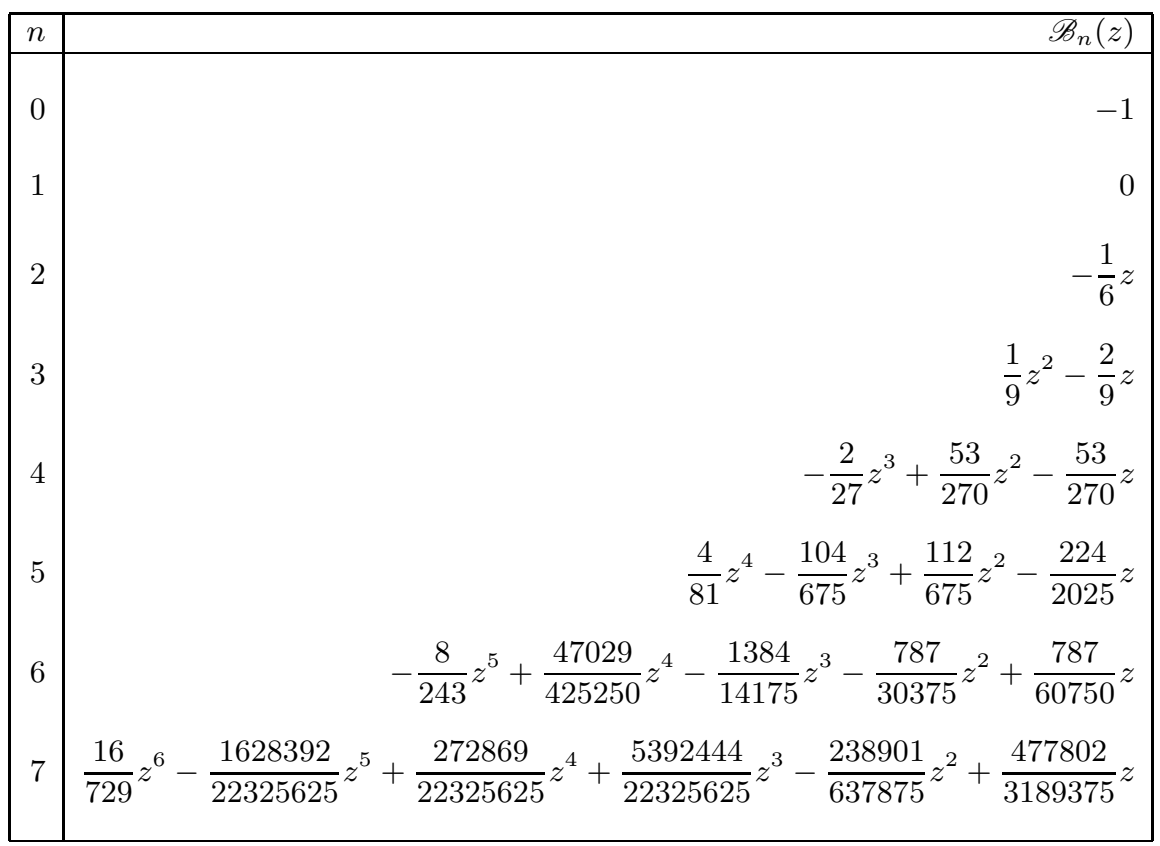

Remark. The constant $\frac{3}{4}$ can be replaced by any constant less than $1.29^{-1}$ (the latter comes exactly from Lemma A.3. . Unfortunately, our method does not allow to prove an absolute convergence in the disk $|z| \leq 1$. In fact, apparently the true region of convergence of the series in question is the half-plane $\Re z \leq 1$. Take, for example, $z_{0}=\frac{2}{3}+4 i$. Then by (6) and the symmetry property one has

$$
\begin{array}{r}
G\left(z_{0}\right)=\frac{1}{2} G\left(z_{0}-1\right)-\frac{1}{2\left(z_{0}-1\right)^{2}} G\left(\frac{1}{z_{0}-1}\right)-\frac{1}{2\left(z_{0}-1\right)} \\
=-\frac{1}{2\left(z_{0}-2\right)^{2}} G\left(\frac{z_{0}-1}{z_{0}-2}\right)-\frac{1}{2\left(z_{0}-1\right)^{2}} G\left(\frac{1}{z_{0}-1}\right)-\frac{1}{2\left(z_{0}-2\right)}-\frac{1}{2\left(z_{0}-1\right)} .
\end{array}
$$

Both arguments under $G$ on the right belong to the unit circle, and thus we can use the Taylor series for $G(z)$. Using numerical values of $m_{L}$, obtained via the method described in Appendix A.2., we obtain: $G\left(z_{0}\right)=0.078083_{+}+0.205424_{+} i$, with all digits exact. On the other hand, the series in Theorem 1.2 for $n=60$ gives

$$
\sum_{n=0}^{60}(-1)^{n} \mathbf{H}_{n}\left(z_{0}\right)=0.078090_{+}+0.205427_{+} i .
$$

Finally, based on the last integral in (5), we can calculate $G(z)$ as a Stieltjes integral. If we divide the unit interval into $N=3560$ equal subintervals, and use the Riemann-Stieltjes sum, we get an approximate value $G\left(z_{0}\right) \approx 0.078082_{+}+$ $0.205424_{+} i$. All evaluations match very well.

Experimental Observation 1.3. We conjecture that the series in Theorem 1.2 converges absolutely for $\Re z \leq 1$.

With a slight abuse of notation, we will henceforth write $f^{(L-1)}\left(z_{0}\right)$ instead of $\left.\frac{\mathrm{d}^{L-1}}{\mathrm{~d} z^{L-1}} f(z)\right|_{z=z_{0}}$. 
Corollary 1.4. The moments $m_{L}$ can be expressed by the convergent series of rational numbers:

$m_{L}=\lim _{n \rightarrow \infty} 2^{2-n} \sum_{a_{1}+a_{2}+\ldots+a_{s}=n}\left[0, a_{1}, a_{2}, \ldots, a_{s}\right]^{L}=\frac{1}{(L-1) !} \sum_{n=0}^{\infty}(-1)^{n} \mathbf{H}_{n}^{(L-1)}(0)$.

The speed of convergence is given by the following estimate: $\left|\mathbf{H}_{n}^{(L-1)}(0)\right| \ll \frac{1}{n^{M}}$, for every $M \in \mathbb{N}$. The implied constant depends only on $L$ and $M$.

Thus, $m_{2}=\sum_{n=0}^{\infty}(-1)^{n} \mathbf{H}_{n}^{\prime}(0)=0.2909264764_{+}$. Regarding the speed, numerical calculations show that in fact the convergence is geometric. Theorem 1.2 in the case $z=1$ gives

$$
M_{1}=G(1)=1+0+\sum_{n=0}^{\infty} \frac{1}{6}\left(\frac{2}{3}\right)^{n}=\frac{3}{2},
$$

which we already know (see Corollary 4.5, the above is a Taylor series for $M_{1}(\mathrm{p})$ in powers of $p-2$, specialized at $\left.p_{0}=1\right)$. Geometric convergence would be the consequence of the fact that analytic functions $m_{L}(\mathrm{p})$ extend beyond $\mathrm{p}=1$ (see below). This is supported by the phenomena represented as Experimental Observation 1.5. Meanwhile, we are able to prove only the given rate. If we were allowed to use the point $z=1$, Theorem 1.2 would give a convergent series for the moments $M_{L}$ as well. This is exactly the same as the series in Corollary 1.4, only one needs to use a point $z=1$ instead of $z=0$.

Experimental Observation 1.5. For $L \geq 1$, the series

$$
M_{L}(\mathrm{p})=\frac{1}{(L-1) !} \cdot \sum_{n=0}^{\infty}(\mathrm{p}-2)^{n} \mathbf{H}_{n}^{(L-1)}(1), \quad M_{L}(1)=M_{L},
$$

has exactly $2-\frac{1}{\sqrt[L]{2}}$ as a radius of convergence.

To this account, Proposition 4.3 endorses this phenomenon, which is highly supported by numerical calculations, and which does hold for $L=1$.

The following three tables give starting values for the sequence $\mathbf{H}_{n}^{\prime}(0)$.

\begin{tabular}{|c|c||c|c|}
\hline$n$ & $\mathbf{H}_{n}^{\prime}(0)$ & $n$ & $\mathbf{H}_{n}^{\prime}(0)$ \\
\hline 0 & $\frac{1}{4}$ & 5 & $-\frac{7}{2 \cdot 3^{4} \cdot 5^{2}}$ \\
1 & 0 & 6 & $-\frac{787}{2^{8} \cdot 3^{5} \cdot 5^{3}}$ \\
2 & $\frac{1}{48}$ & 7 & $\frac{238901}{2^{7} \cdot 3^{6} \cdot 5^{4} \cdot 7}$ \\
3 & $-\frac{1}{72}$ & 8 & $-\frac{181993843}{2^{10} \cdot 3^{7} \cdot 5^{5} \cdot 7^{2}}$ \\
4 & $\frac{53}{8640}$ & 9 & $\frac{12965510861}{2^{6} \cdot 3^{8} \cdot 5^{6} \cdot 7^{3} \cdot 17}$ \\
\hline
\end{tabular}




\begin{tabular}{|c|c|}
\hline$n$ & $\mathbf{H}_{n}^{\prime}(0)$ \\
\hline 10 & $-\frac{8026531718888633}{2^{12} \cdot 3^{9} \cdot 5^{7} \cdot 7^{4} \cdot 11 \cdot 17^{2}}$ \\
11 & $\frac{797209536976557079423}{2^{11} \cdot 3^{10} \cdot 5^{8} \cdot 7^{5} \cdot 11^{2} \cdot 17^{3} \cdot 31}$ \\
12 & $\frac{4198988799919158293319845971}{2^{14} \cdot 3^{11} \cdot 5^{9} \cdot 7^{6} \cdot 11^{3} \cdot 13 \cdot 17^{4} \cdot 31^{2}}$ \\
13 & $\frac{12702956822417247965298252330349561}{2^{10} \cdot 3^{12} \cdot 5^{10} \cdot 7^{7} \cdot 11^{4} \cdot 13^{2} \cdot 17^{5} \cdot 31^{3}}$ \\
& $\frac{7226191636013675292833514548603516395499899}{2^{16} \cdot 3^{13} \cdot 5^{11} \cdot 7^{8} \cdot 11^{5} \cdot 13^{3} \cdot 17^{6} \cdot 31^{4}}$ \\
\hline
\end{tabular}

\begin{tabular}{|c|c|}
\hline$n$ & $\mathbf{H}_{n}^{\prime}(0)$ \\
\hline 15 & $-\frac{129337183009042141853748450730581369733226857443915617}{2^{15} \cdot 3^{14} \cdot 5^{12} \cdot 7^{9} \cdot 11^{6} \cdot 13^{4} \cdot 17^{7} \cdot 31^{5} \cdot 43 \cdot 127}$ \\
16 & $\frac{31258186275777197041073243752715109842753785598306812028984213251}{2^{18} \cdot 3^{15} \cdot 5^{13} \cdot 7^{10} \cdot 11^{7} \cdot 13^{5} \cdot 17^{8} \cdot 31^{6} \cdot 43^{2} \cdot 127^{2}}$ \\
17 & $-\frac{3282520501229639755997762022707321704397776888948469860959830459774414444483}{2^{12} \cdot 3^{16} \cdot 5^{14} \cdot 7^{11} \cdot 11^{8} \cdot 13^{6} \cdot 17^{9} \cdot 31^{7} \cdot 43^{3} \cdot 127^{3} \cdot 257}$ \\
\hline
\end{tabular}

The float values of the last three rational numbers are -0.000025804822076 , 0.000018040274062 and -0.000010917558446 , respectively. The alternating sum of the elements in the table is $\sum_{n=0}^{N}(-1)^{n} \mathbf{H}_{n}^{\prime}(0)=0.2909255862_{+}$(where $N=17$ ), whereas $N=40$ gives $0.2909264880_{+}$, and $N=50$ gives $0.2909264784_{+}$. Note that the manifestation of Fermat and Mersenne primes in the denominators at an early stage is not accidental, minding the exact value of the determinant in Lemma 6.1. Chapter 6 (see below). Moreover, the prime powers of every odd prime that divides the denominator increase each time by 1 while passing from $\mathbf{H}_{n}^{\prime}(0)$ to $\mathbf{H}_{n+1}^{\prime}(0)$. The pattern for the powers of 2 is more complicated. More thorough research of the linear map in Lemma 6.1 can thus clarify prime decomposition of denominators; numerators remains much more complicated.

As will be apparent later, the result in Theorem 1.2 is derived from the knowledge of $\mathrm{p}$-derivatives of $G(\mathrm{p}, z)$ at $\mathrm{p}=2$ (see below). On the other hand, since there are two points $(\mathrm{p}=2$ and $\mathrm{p}=0)$ such that all higher $\mathrm{p}$-derivatives of $G(\mathrm{p}, z)$ are rational functions in $z$, it is not completely surprising that the approach through $\mathrm{p}=0$ also gives convergent series for the moments, though in this case we are forced to use Borel summation. At this point, the author does not have a strict mathematical proof of this result (since the function $G(\mathrm{p}, z)$ is meanwhile defined only for $\Re p \geq 1$ ), though numerical calculations provide overwhelming evidence for its validity. 
Experimental Observation 1.6. Define the rational functions (with rational coefficients) $\mathbf{Q}_{n}(z), n \geq 0$, by

$$
\begin{aligned}
& \mathbf{Q}_{0}(z)=-\frac{1}{2 z}, \text { and recurrently by } \\
& \mathbf{Q}_{n}(z)=\frac{1}{2} \sum_{j=0}^{n-1} \frac{1}{j !} \cdot \frac{\partial^{j}}{\partial z^{j}} \mathbf{Q}_{n-j-1}(-1) \cdot\left(z^{j}-\frac{1}{z^{j+2}}\right) .
\end{aligned}
$$

Then

$$
\begin{aligned}
m_{L} & =\lim _{n \rightarrow \infty} 2^{2-n} \sum_{a_{1}+a_{2}+\ldots+a_{s}=n}\left[0, a_{1}, a_{2}, \ldots, a_{s}\right]^{L} \\
& =\frac{1}{(L-1) !} \sum_{r=0}^{\infty}\left(\sum_{n=0}^{\infty} \frac{\mathbf{Q}_{n}^{(L-1)}(-1)}{n !} \cdot \int_{r}^{r+1} t^{n} e^{-t} \mathrm{~d} t\right) .
\end{aligned}
$$

Moreover,

$$
\mathbf{Q}_{n}(z)=\frac{(z+1)(z-1) \mathscr{D}_{n}(z)}{z^{n+1}}, \quad n \geq 1,
$$

where $\mathscr{D}_{n}(z)$ are polynomials with rational coefficients $\left(\mathbb{Q}_{p}\right.$ integers for $\left.p \neq 2\right)$ of degree $2 n-2$ with the reciprocity property

$$
\mathscr{D}_{n}(z)=z^{2 n-2} \mathscr{D}_{n}\left(\frac{1}{z}\right) .
$$

Note the order of summation in the series for $m_{L}$, since the reason for introducing exponential functions is because we use Borel summation. For example,

$$
" 1-2+4-8+16-32+\ldots " \stackrel{\text { Borel }}{=} \sum_{r=0}^{\infty}\left(\sum_{n=0}^{\infty} \frac{(-2)^{n}}{n !} \cdot \int_{r}^{r+1} t^{n} e^{-t} \mathrm{~d} t\right)=\frac{1}{3} .
$$

The following table gives initial results.

\begin{tabular}{|c|l||c|l|}
\hline$n$ & $\mathscr{D}_{n}(z)$ & $n$ & $\mathscr{D}_{n}(z)$ \\
\hline 1 & $\frac{1}{4}$ & 4 & $\frac{1}{8}\left(2 z^{6}-3 z^{5}+6 z^{4}-3 z^{3}+6 z^{2}-3 z+2\right)$ \\
2 & $\frac{1}{4}\left(z^{2}+1\right)$ & 5 & $\frac{1}{4}\left(z^{8}-2 z^{7}+4 z^{6}-7 z^{5}+4 z^{4}-7 z^{3}+4 z^{2}-2 z+1\right)$ \\
3 & $\frac{1}{4}\left(z^{4}-z^{3}+z^{2}\right.$ & 6 & $\frac{1}{8}\left(2 z^{10}-5 z^{9}+12 z^{8}-20 z^{7}+37 z^{6}\right.$ \\
& \multicolumn{1}{|c|}{$-z+1)$} & & $\left.-20 z^{5}+37 z^{4}-20 z^{3}+12 z^{2}-5 z+2\right)$ \\
\hline
\end{tabular}

The next table gives $\mathbf{Q}_{n}^{\prime}(-1)=2(-1)^{n} \mathscr{D}_{n}(-1)$ explicitly: these constants appear in the series defining the first nontrivial moment $m_{2}$. Also, since these numbers are $p$-adic integers for $p \neq 2$, there is a hope for the successful implementation of the idea from the last chapter in [2] that is, possibly one can define moments $m_{L}$ as $p$-adic rationals as well. 


\begin{tabular}{|rr||rr||rr||cc|}
\hline$n$ & $\mathbf{Q}_{n}^{\prime}(-1)$ & $n$ & $\mathbf{Q}_{n}^{\prime}(-1)$ & $n$ & $\mathbf{Q}_{n}^{\prime}(-1)$ & $n$ & $\mathbf{Q}_{n}^{\prime}(-1)$ \\
\hline 0 & $\frac{1}{2}$ & 8 & $\frac{1417}{4}$ & 16 & $\frac{206836175}{64}$ & 24 & $\frac{1685121707817}{32}$ \\
1 & $-\frac{1}{2}$ & 9 & $-\frac{8431}{8}$ & 17 & $-\frac{339942899}{32}$ & 25 & $-\frac{92779913448103}{512}$ \\
2 & 1 & 10 & $\frac{50899}{16}$ & 18 & $\frac{1125752909}{32}$ & 26 & $\frac{80142274019997}{128}$ \\
3 & $-\frac{5}{2}$ & 11 & -9751 & 19 & $-\frac{15014220659}{128}$ & 27 & $-\frac{1111839248032133}{512}$ \\
4 & $\frac{25}{4}$ & 12 & 30365 & 20 & $\frac{25188552721}{64}$ & 28 & $\frac{7740056893342455}{1024}$ \\
5 & -16 & 13 & $-\frac{3069719}{32}$ & 21 & $-\frac{170016460947}{128}$ & 29 & $-\frac{13515970598654393}{512}$ \\
6 & 43 & 14 & $\frac{1227099}{4}$ & 22 & $\frac{1153784184807}{256}$ & 30 & $\frac{47354245650630005}{512}$ \\
7 & $-\frac{971}{8}$ & 15 & $-\frac{31719165}{32}$ & 23 & $-\frac{983668214037}{64}$ & 31 & $-\frac{665632101181145115}{2048}$ \\
\hline
\end{tabular}

The final table in this section lists float values of the constants

$$
\vartheta_{r}=\sum_{n=0}^{\infty} \frac{\mathbf{Q}_{n}^{\prime}(-1)}{n !} \cdot \int_{r}^{r+1} t^{n} e^{-t} \mathrm{~d} t, \quad r \in \mathbb{N}_{0}, \quad \sum_{r=0}^{\infty} \vartheta_{r}=m_{2},
$$

appearing in the Borel summation.

\begin{tabular}{|r|r||r|r|}
\hline$r$ & $\vartheta_{r}$ & $r$ & $\vartheta_{r}$ \\
\hline 0 & 0.2327797875 & 6 & 0.0004701146 \\
1 & 0.0471561089 & 7 & 0.0004980015 \\
2 & 0.0085133626 & 8 & 0.0004005270 \\
3 & 0.0005892453 & 9 & 0.0002722002 \\
4 & -0.0001872357 & 10 & 0.0001607897 \\
5 & 0.0002058729 & 11 & 0.0000812407 \\
\hline
\end{tabular}

Thus, $\sum_{r=0}^{11} \vartheta_{r}=0.2909400155_{+}=m_{2}+0.000013539_{+}$.

This paper is organized as follows. In Section 2, for each $p, 1 \leq p<\infty$, we introduce a generalization of the Farey (Calkin-Wilf) tree, denoted by $\mathcal{Q}_{\mathrm{p}}$. This leads to the notion of $\mathrm{p}$-continued fractions and $\mathrm{p}$-Minkowski question mark functions $F_{\mathrm{p}}(x)$. Though p-continued fractions are of independent interest (one could define a transfer operator, to prove an analogue of the Gauss-Kuzmin-Lévy theorem, various metric results and introduce structural constants), we confine ourselves to the facts which are necessary for our purposes and leave the deeper research for the future. In Section 3 we extend these results to the case of complex $\mathrm{p},|\mathrm{p}-2| \leq 1$. The crucial consequence of these results is the fact that a function $\mathfrak{X}(\mathrm{p}, x)$ (which gives a bijection between trees $\mathcal{Q}_{1}$ and $\mathcal{Q}_{\mathrm{p}}$ ) is a continuous function in $x$ and an analytic function in $\mathrm{p}$ for $|\mathrm{p}-2| \leq 1$. In Section 4 we introduce exactly the same integral transforms of $F_{\mathrm{p}}(x)$ as was done in a special (though most important) case of $F(x)=F_{1}(x)$. Also, in this section we prove certain relations among the moments. In Section 5 we give the proof of the three-term functional equation for $G_{\mathrm{p}}(z)$ and the integral equation for $\mathfrak{m}_{\mathrm{p}}(t)$. Finally, Theorem 1.2 is proved in Section 6. The hierarchy of sections is linear, and all results from previous sections are used in Section 6. Appendix A contains: derivation for the series (7); MAPLE codes to compute rational functions $\mathbf{H}_{n}(z)$ and $\mathbf{Q}_{n}(z)$; description of a high-precision method to calculate numerical values for the constants $m_{L}$; auxiliary lemmas for Section 3. The paper also contains graphs of some p-Minkowski question mark functions $F_{\mathrm{p}}(x)$ for real $\mathrm{p}$, and also pictures of locus points of elements of trees $\mathcal{Q}_{\mathrm{p}}$ for certain characteristic values of $\mathrm{p}$. 


\section{2. p-QUESTION MARK FUNCTIONS AND p-CONTINUED FRACTIONS}

In this section we introduce a family of natural generalizations of the Minkowski question mark function $F(x)$. Let $1 \leq \mathrm{p}<2$. Consider the following binary tree, which we denote by $\mathcal{Q}_{\mathrm{p}}$. We start from the root $x=1$. Further, each element ("root") $x$ of this tree generates two "offspring" by the following rule:

$$
x \mapsto \frac{\mathrm{p} x}{x+1}, \quad \frac{x+1}{\mathrm{p}} .
$$

We will use the notation $\mathcal{T}_{\mathrm{p}}(x)=\frac{x+1}{\mathrm{p}}, \mathcal{U}_{\mathrm{p}}(x)=\frac{\mathrm{p} x}{x+1}$. When $\mathrm{p}$ is fixed, we will sometimes discard the subscript. Thus, the first four generations lead to (8)

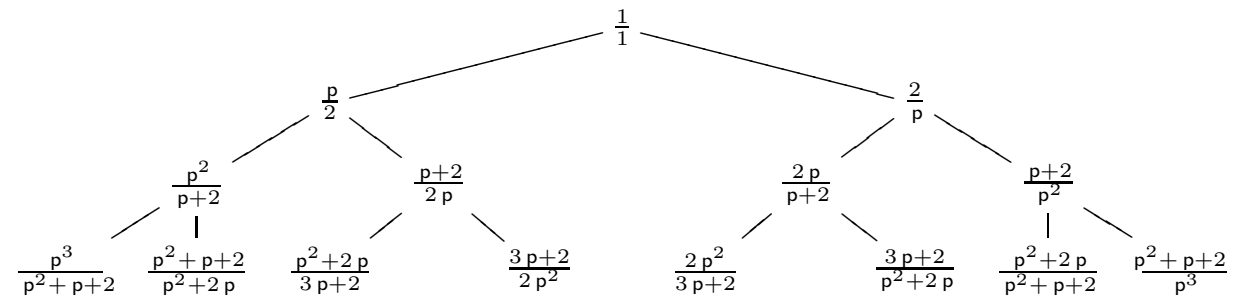

We refer the reader to the paper [11, where authors consider a rather similar construction, though having a different purpose in mind (see also [6]). Denote by $T_{n}(\mathrm{p})$ the sequence of polynomials, appearing as numerators of fractions of this tree. Thus, $T_{1}(\mathrm{p})=1, T_{2}(\mathrm{p})=\mathrm{p}, T_{3}(\mathrm{p})=2$. Directly from the definition of this tree we inherit that

$$
\begin{aligned}
T_{2 n}(\mathrm{p}) & =\mathrm{p} T_{n}(\mathrm{p}) \text { for } n \geq 1, \\
T_{2 n-1}(\mathrm{p}) & =T_{n-1}(\mathrm{p})+\mathrm{p}^{-\epsilon} T_{n}(\mathrm{p}) \text { for } n \geq 2,
\end{aligned}
$$

where $\epsilon=\epsilon(n)=1$ if $n$ is a power of two, and $\epsilon=0$ otherwise. Thus, the definition of these polynomials is almost the same as it appeared in [17] (these polynomials were named Stern polynomials by the authors), with the distinction that in [17] everywhere one has $\epsilon=0$. Naturally, this difference produces different sequences of polynomials.

There are $2^{n-1}$ positive real numbers in each generation of the tree $\mathcal{Q}_{\mathrm{p}}$, say $a_{k}^{(n)}$, $1 \leq k \leq 2^{n-1}$. Moreover, they are all contained in the interval $\left[\mathrm{p}-1, \frac{1}{\mathrm{p}-1}\right]$. Indeed, this holds for the initial root $x=1$, and

$$
\begin{aligned}
& \mathrm{p}-1 \leq x \leq \frac{1}{\mathrm{p}-1} \Leftrightarrow \mathrm{p}-1 \leq \frac{\mathrm{p} x}{x+1} \leq 1, \\
& \mathrm{p}-1 \leq x \leq \frac{1}{\mathrm{p}-1} \Leftrightarrow 1 \leq \frac{x+1}{\mathrm{p}} \leq \frac{1}{\mathrm{p}-1} .
\end{aligned}
$$

This also shows that the left offspring is contained in the interval $[p-1,1]$, while the right one is in the interval $\left[1, \frac{1}{p-1}\right]$. The real numbers appearing in this tree have an intrinsic relation with the $\mathrm{p}$-continued fractions algorithm. The definition of the latter is as follows. Let $x \in\left(\mathrm{p}-1, \frac{1}{\mathrm{p}-1}\right)$. Consider the following procedure:

$$
R_{\mathrm{p}}(x)=\left\{\begin{array}{cl}
\mathcal{T}^{-1}(x)=\mathrm{p} x-1, & \text { if } 1 \leq x<\frac{1}{\mathrm{p}-1} \\
\mathcal{I}(x)=\frac{1}{x}, & \text { if } \mathrm{p}-1<x<1 \\
\text { STOP }, & \text { if } x=\mathrm{p}-1
\end{array}\right.
$$


Then each such $x$ can be uniquely represented as a $\mathrm{p}$-continued fraction

$$
x=\left[a_{0}, a_{1}, a_{2}, a_{3}, \ldots .\right]_{\mathrm{p}},
$$

where $a_{i} \in \mathbb{N}$ for $i \geq 1$, and $a_{0} \in \mathbb{N} \cup\{0\}$. This notation means that in the course of the iterations $R_{\mathrm{p}}^{\infty}(x)$ we apply $\mathcal{T}^{-1}(x)$ exactly $a_{0}$ times, then $\mathcal{I}$ once, then we apply $\mathcal{T}^{-1}$ exactly $a_{1}$ times, then $\mathcal{I}$, and so on. The procedure terminates exactly for those $x \in\left(\mathrm{p}-1, \frac{1}{\mathrm{p}-1}\right)$ that are the members of the tree $\mathcal{Q}_{\mathrm{p}}$ ("p-rationals"). Also, direct inspection shows that if the procedure does terminate, the last entry $a_{s} \geq 2$. Thus, we have the same ambiguity for the last entry exactly as is the case with ordinary continued fractions. At this point it is straightforward to show that the $n$th generation of $\mathcal{Q}_{\mathrm{p}}$ consists of $x=\left[a_{0}, a_{1}, \ldots, a_{s}\right]_{\mathrm{p}}$ such that $\sum_{j=0}^{s} a_{j}=n$, exactly as in the case $\mathrm{p}=1$ and the tree (2).

Now, consider a function $\mathfrak{X}_{\mathrm{p}}(x)$ with the following property: $\mathfrak{X}_{\mathrm{p}}(x)=\bar{x}$, where $x$ is a rational number in the Calkin-Wilf tree (2), and $\bar{x}$ is a corresponding number in the tree (8). In other words, $\mathfrak{X}_{\mathrm{p}}(x)$ is simply a bijection between these two trees. First, if $x<y$, then $\bar{x}<\bar{y}$. Also, all positive rationals appear in the tree (2) and they are everywhere dense in $\mathbb{R}_{+}$. Moreover, $\mathcal{T}$ and $\mathcal{U}$ both preserve order, and $\left[\mathrm{p}-1, \frac{1}{\mathrm{p}-1}\right)$ is a disjoint union of $\mathcal{T}\left[\mathrm{p}-1, \frac{1}{\mathrm{p}-1}\right)$ and $\mathcal{U}\left[\mathrm{p}-1, \frac{1}{\mathrm{p}-1}\right)$. Now it is obvious that the function $\mathfrak{X}_{\mathrm{p}}(x)$ can be extended to a continuous monotone increasing function

$$
\mathfrak{X}_{\mathrm{p}}(\star): \mathbb{R}_{+} \rightarrow\left[\mathrm{p}-1, \frac{1}{\mathrm{p}-1}\right), \quad \mathfrak{X}_{\mathrm{p}}(\infty)=\frac{1}{\mathrm{p}-1} .
$$

Thus,

$$
\mathfrak{X}_{\mathrm{p}}\left(\left[a_{0}, a_{1}, a_{2}, a_{3} \ldots\right]\right)=\left[a_{0}, a_{1}, a_{2}, a_{3} \ldots\right]_{\mathrm{p}} .
$$

As can be seen from the definitions of both trees (2) and (8), this function satisfies the functional equations

$$
\begin{aligned}
\mathfrak{X}_{\mathrm{p}}(x+1) & =\frac{\mathfrak{X}_{\mathrm{p}}(x)+1}{\mathrm{p}}, \\
\mathfrak{X}_{\mathrm{p}}\left(\frac{x}{x+1}\right) & =\frac{\mathrm{p} \mathfrak{X}_{\mathrm{p}}(x)}{\mathfrak{X}_{\mathrm{p}}(x)+1}, \\
\mathfrak{X}_{\mathrm{p}}\left(\frac{1}{x}\right) & =\frac{1}{\mathfrak{X}_{\mathrm{p}}(x)} .
\end{aligned}
$$

The last one (symmetry property) is a consequence of the first two. We are not aware whether this notion of p-continued fractions is new or not. For example,

$$
\begin{aligned}
\frac{1+\sqrt{1+4 \mathrm{p}}}{2 \mathrm{p}} & =[1,1,1,1,1,1, \ldots]_{\mathrm{p}}=\mathfrak{X}_{\mathrm{p}}\left(\frac{1+\sqrt{5}}{2}\right), \\
\sqrt{3} & =[4,2,1,10,1,1,2,1,5,1,1,2,1,2,1,1,2,1,3,7,4, \ldots]_{\frac{3}{2}}, \\
2 & =[4,1,1, \overline{2,1,1}]_{\sqrt{2}} .
\end{aligned}
$$

Now fix $\mathrm{p}, 1 \leq \mathrm{p}<2$. The following proposition follows immediately from the properties of $F(x)$.

Proposition 2.1. There exists a limit distribution of the $n$th generation of the tree $\mathcal{Q}_{\mathrm{p}}$ as $n \rightarrow \infty$, defined as

$$
F_{\mathrm{p}}(x)=\lim _{n \rightarrow \infty} 2^{-n+1} \#\left\{k: a_{k}^{(n)}<x\right\} .
$$


This function is continuous, $F_{\mathrm{p}}(x)=0$ for $x \leq \mathrm{p}-1, F_{\mathrm{p}}(x)=1$ for $x \geq \frac{1}{\mathrm{p}-1}$, and it satisfies two functional equations:

$$
2 F_{\mathrm{p}}(x)=\left\{\begin{array}{cl}
F_{\mathrm{p}}(\mathrm{p} x-1)+1, & \text { if } 1 \leq x \leq \frac{1}{\mathrm{p}-1}, \\
F_{\mathrm{p}}\left(\frac{x}{\mathrm{p}-x}\right), & \text { if } \mathrm{p}-1 \leq x \leq 1 .
\end{array}\right.
$$

Additionally,

$$
F_{\mathrm{p}}(x)+F_{\mathrm{p}}\left(\frac{1}{x}\right)=1 \text { for } x>0 .
$$

The explicit expression for $F_{\mathrm{p}}(x)$ is given by

$$
F_{\mathrm{p}}\left(\left[a_{0}, a_{1}, a_{2}, a_{3}, \ldots\right]_{\mathrm{p}}\right)=1-2^{-a_{0}}+2^{-\left(a_{0}+a_{1}\right)}-2^{-\left(a_{0}+a_{1}+a_{2}\right)}+\ldots
$$

We will refer to the last functional equation as the symmetry property. As was said, it is a consequence of the other two, though it is convenient to separate it.

Proof. Indeed, as is obvious from the observations above, we simply have

$$
F_{\mathrm{p}}\left(\mathfrak{X}_{\mathrm{p}}(x)\right)=F(x), \quad x \in[0, \infty) .
$$

Therefore, two functional equations follow from (3) and (9). All the other statements are immediate and follow from the properties of $F(x)$.

Equally important, consider the binary tree (8) for $\mathrm{p}>2$. In this case an analogous proposition holds.

Proposition 2.2. Let $\mathrm{p}>2$. Then there exists a limit distribution of the $n$th generation as $n \rightarrow \infty$. Denote it by $f_{\mathrm{p}}(x)$ This function is continuous, $f_{\mathrm{p}}(x)=0$ for $x \leq \frac{1}{\mathrm{p}-1}, f_{\mathrm{p}}(x)=1$ for $x \geq \mathrm{p}-1$, and it satisfies two functional equations:

$$
2 f_{\mathrm{p}}(x)=\left\{\begin{array}{cl}
f_{\mathrm{p}}(\mathrm{p} x-1) & \text { if } 1 \leq x \leq \mathrm{p}-1 \\
f_{\mathrm{p}}\left(\frac{x}{\mathrm{p}-x}\right)+1 & \text { if } \frac{1}{\mathrm{p}-1} \leq x \leq 1
\end{array}\right.
$$

and

$$
f_{\mathrm{p}}(x)+f_{\mathrm{p}}\left(\frac{1}{x}\right)=1 \text { for } x>0 .
$$

Proof. The proof is analogous to that of Proposition 2.1, only this time we use equivalences

$$
\begin{aligned}
& \mathrm{p}-1 \leq x \leq \frac{1}{\mathrm{p}-1} \Leftrightarrow 1 \leq \frac{\mathrm{p} x}{x+1} \leq \mathrm{p}-1, \\
& \mathrm{p}-1 \leq x \leq \frac{1}{\mathrm{p}-1} \Leftrightarrow \frac{1}{\mathrm{p}-1} \leq \frac{x+1}{\mathrm{p}} \leq \mathrm{p}-1 .
\end{aligned}
$$

For the sake of uniformity, we introduce $F_{\mathrm{p}}(x)=1-f_{\mathrm{p}}(x)$ for $\mathrm{p}>2$. Then $F_{\mathrm{p}}(x)$ satisfies exactly the same functional equations (3) , with a slight difference that $F_{\mathrm{p}}(x)=1$ for $x \leq \frac{1}{\mathrm{p}-1}$ and $F_{\mathrm{p}}(x)=0$ for $x \geq \mathrm{p}-1$. Consequently, we will not separate these two cases and all our subsequent results hold uniformly. To this account it should be noted that, for example, in case $\mathrm{p}>2$ the integral $\int_{\mathrm{p}-1}^{1} \star d \star$ should be understood as $-\int_{1}^{\mathrm{p}-1} \star \mathrm{d} \star$. Figure 1 gives graphic images of typical cases for $F_{\mathrm{p}}(x)$. 

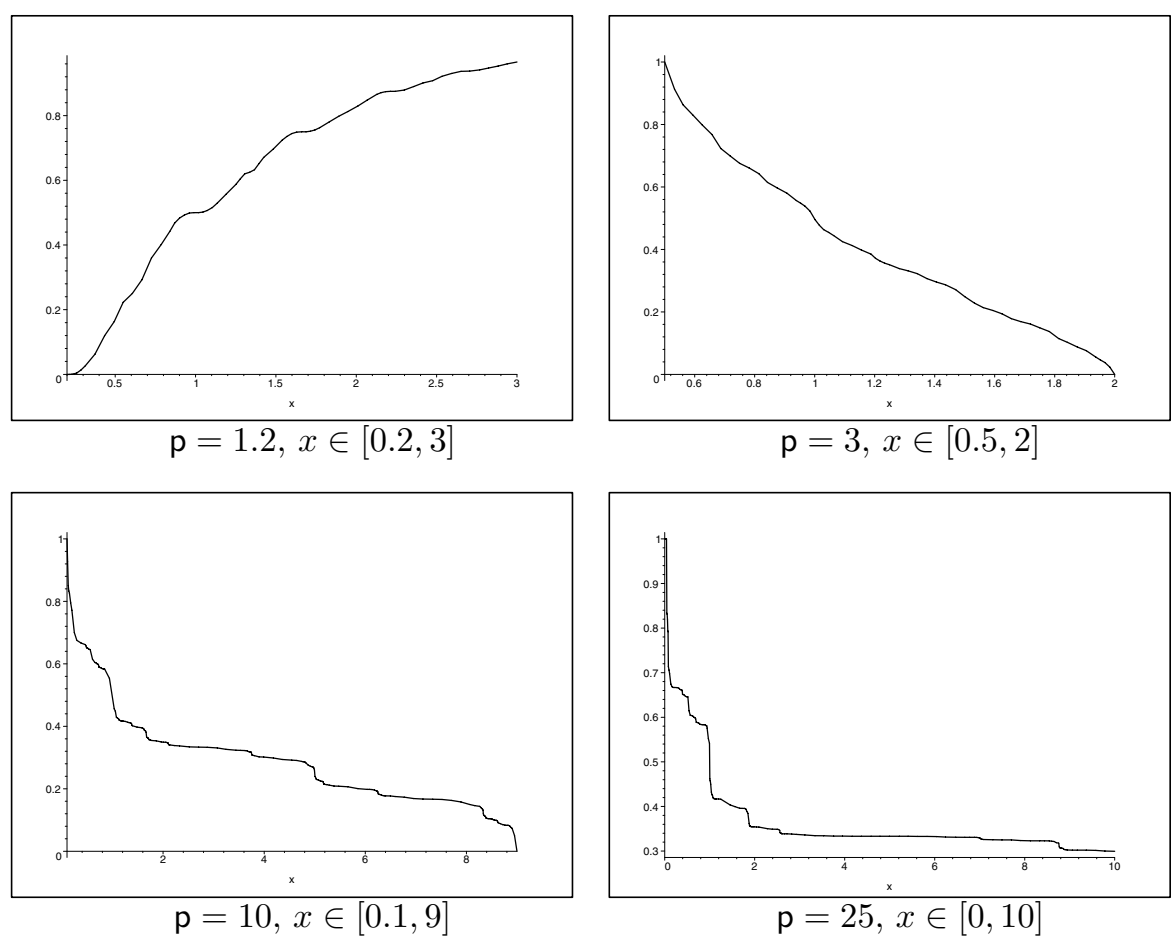

FiguRe 1. Functions $F_{\mathrm{p}}(x)$

\section{Complex case}

After dealing with the case of real $\mathrm{p}, 1 \leq \mathrm{p}<\infty$, let us consider a tree (8) when $p \in \mathbb{C}$. For our purpose we will concentrate on the case $|p-2| \leq 1$. It should be noted that the method which we use allows us to extend these results to the case $\Re p \geq 1$. The question of determining the set in the complex plane where similar results are valid remains open. More importantly, the problem of determining all $\mathrm{p} \in \mathbb{C}$ for which there exists an analytic function $G_{\mathrm{p}}(z)$, which satisfies (22), seems to be much harder and more interesting. Here and below $[0, \infty]$ stands for a compactification of $[0, \infty)$. In the sequel, the notion of a function $f(z)$ to be analytic in the closed disc $|z-2| \leq 1$ means that for $z_{0} \neq 1,\left|z_{0}-2\right| \leq 1$, this function is analytic in a certain small neighborhood of $z_{0}$. If $z_{0}=1$, this means that there exist all higher derivatives if one approaches the point $z_{0}=1$ while remaining in the disc $|z-2| \leq 1$.

In this section we prove the following result.

Theorem 3.1. There exists a unique function $\mathfrak{X}_{\mathrm{p}}(x)=\mathfrak{X}(\mathrm{p}, x):\{|\mathrm{p}-2| \leq 1\}$ $\times\{[0, \infty]\} \rightarrow \mathbb{C} \cup\{\infty\}$, having these properties:

(i) $\mathfrak{X}(\mathrm{p}, x)$ satisfies the functional equations (9).

(ii) For fixed $\mathrm{p} \neq 1, \mathfrak{X}(\mathrm{p}, x):[0, \infty] \rightarrow \mathbb{C}$ is a continuous function, and the image (denote it by $\mathscr{I}_{\mathrm{p}}$ ) is thus a bounded curve; it is contained in the domain $\mathbb{C} \backslash\left\{|z+1| \leq \frac{3}{4}\right\}$.

(iii) For every $\mathrm{p},|\mathrm{p}-2| \leq 1, \mathrm{p} \neq 1$, in some neighborhood of $\mathrm{p}$ there exists the derivative $\frac{\partial}{\partial \mathrm{p}} \mathfrak{X}(\mathrm{p}, x)$, which is a continuous function for $x \in[0, \infty]$. 
(iv) There exist all derivatives $\mathcal{S}_{N}(x)=\left.\frac{\partial^{N}}{\partial \mathrm{p}^{N}} \mathfrak{X}(\mathrm{p}, x)\right|_{\mathrm{p}=1}:[0, \infty) \rightarrow \mathbb{R}$ (the derivatives are taken inside $|\mathrm{p}-2| \leq 1)$. These functions are uniformly continuous for irrational $x$ in any finite interval. Moreover, $\mathcal{S}_{N}(x) \ll_{N} x^{N+1}$ for $x \geq 1$, and $\mathcal{S}_{N}(x) \ll_{N} 1$ for $x \in(0,1)$.

The curve $\mathscr{I}_{\mathrm{p}}$ has a natural fractal structure: it decomposes into two parts, namely $\frac{\mathscr{I}_{\mathrm{p}}+1}{\mathrm{p}}$ and $\frac{\mathrm{p} \mathscr{I}_{\mathrm{p}}}{\mathscr{I}_{\mathrm{p}}+1}$, with a common point $z=1$. Additionally, $\mathscr{I}_{\mathrm{p}}=\frac{1}{\mathscr{I}_{\mathrm{p}}}$. As a consequence, $0 \notin \mathscr{I}_{\mathrm{p}}$ for $\mathrm{p} \neq 1$. Figures $2-4$ show the images of $\mathscr{I}_{\mathrm{p}}$ for certain characteristic values of $\mathrm{p}$. They are indeed all continuous curves, at least for $\Re \mathrm{p} \geq 1$ ! Further, Figure 5 shows the image of the curve $\left.\frac{\mathrm{d}}{\mathrm{d} \mathrm{p}} \mathfrak{X}(\mathrm{p}, x)\right|_{\mathrm{p}=1.5+0.5 i}$, $x \in[0, \infty]$.

The investigations of the tree $\mathcal{Q}_{\mathrm{p}}$ deserve a separate paper. I am very grateful to my colleagues Jeffrey Lagarias and Stefano Isola, who sent me various references, also informing me about the intrinsic relations of this problem with: Julia sets of rational maps of the Riemann sphere; iterated function systems; forward limit sets of semigroups; various topics from complex dynamics and the geometry of discrete groups. Thus, the problem is much more subtle and involved than it appears to be. This poses a difficult question on the limit set of the semigroup generated by the transformations $\mathcal{U}_{\mathrm{p}}$ and $\mathcal{T}_{\mathrm{p}}$, or any other two "conjugate" analytic maps of the Riemann sphere (say, two analytic maps $\mathcal{A}$ and $\mathcal{B}$ are "conjugate" if $\mathcal{A}(\alpha)=\alpha$, $\mathcal{B}(\beta)=\beta, \mathcal{A}(\beta)=\mathcal{B}(\alpha)$ for some two points $\alpha$ and $\beta$ on the Riemann sphere). Possibly, certain techniques from complex dynamics do apply here. As pointed out by Curtis McMullen, the property of boundedness of $\mathscr{I}_{\mathrm{p}}$ can be reformulated in a coordinate-free manner. It appears that this curve consists of the closure of the attracting fixed points of the elements of the semigroup $\left\langle\mathcal{T}_{\mathrm{p}}, \mathcal{U}_{\mathrm{p}}\right\rangle$. Then the property for the curve being bounded and being bounded away from $z=0$ means that it does not contain a repelling fixed point of $\mathcal{T}_{\mathrm{p}}(z=\infty)$ and a repelling fixed point of $\mathcal{U}_{\mathrm{p}}(z=0)$. It contains neither of the repelling fixed points of the elements of this semigroup. Note that $\mathcal{T}_{2}(1)=\mathcal{U}_{2}(1)=1, \mathcal{T}_{2}^{\prime}(1)=\mathcal{U}_{2}^{\prime}(1)=1 / 2$. Thus, there exists a small open ball $\mathbf{D}$ around $z=1$, such that $\mathcal{T}_{2}(\mathbf{D}) \subset \mathbf{D}, \mathcal{U}_{2}(\mathbf{D}) \subset \mathbf{D}$, and the last two maps are contractions in $\mathbf{D}$. This strict containment is an open condition on $p$, and thus there exists a neighborhood of $p=2$ such that Theorem 3.1 does hold. I am grateful to Curtis McMullen for this remark: we get the result almost for free. Yet, the full result for $|p-2| \leq 1$ is needed. This is not a new kind of problem. Some cases of pairs of Möbius transformations were studied. For example, the author in 7] deals with the case of a semigroup generated by two maps $z \mapsto s z \pm 1$, for fixed $s,|s|<1$, and investigates a closure of a set of all attracting fixed points. For example, for $|s|>2^{-1 / 2}$, this set is connected. Further development of this problem can be seen in 32]. On the other hand, the case of one rational map is rather well understood, and it is treated in [4]. Thus, though the machinery of complex dynamics can greatly clarify our understanding of the structure of the curve $\mathscr{I}_{\mathrm{p}}$, we will rather employ the techniques from the analytic theory of continued fractions. The main source is the monograph by H.S. Wall [34]. (Lemmas A.1 A.2 and A.3 can be found in Appendix A.2.) 


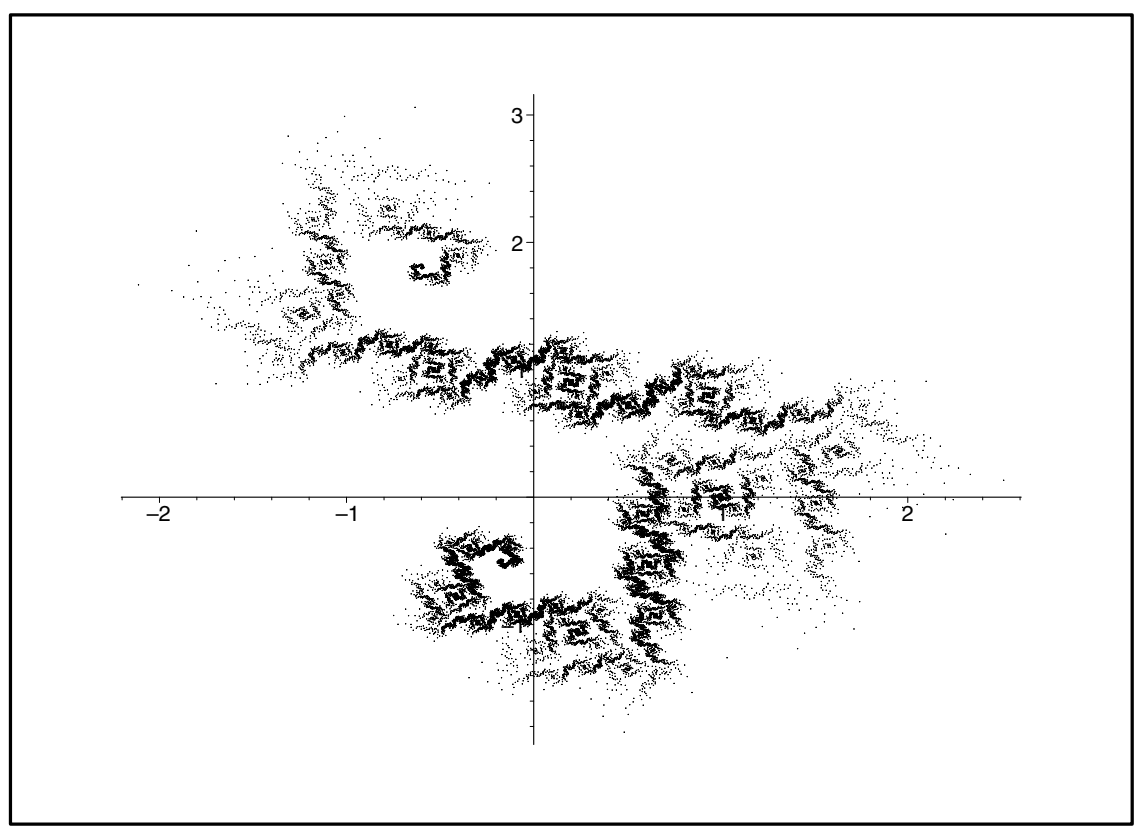

FiguRE $2 . \mathscr{I}_{\mathrm{p}}, \mathrm{p}=0.4+1.8 i$

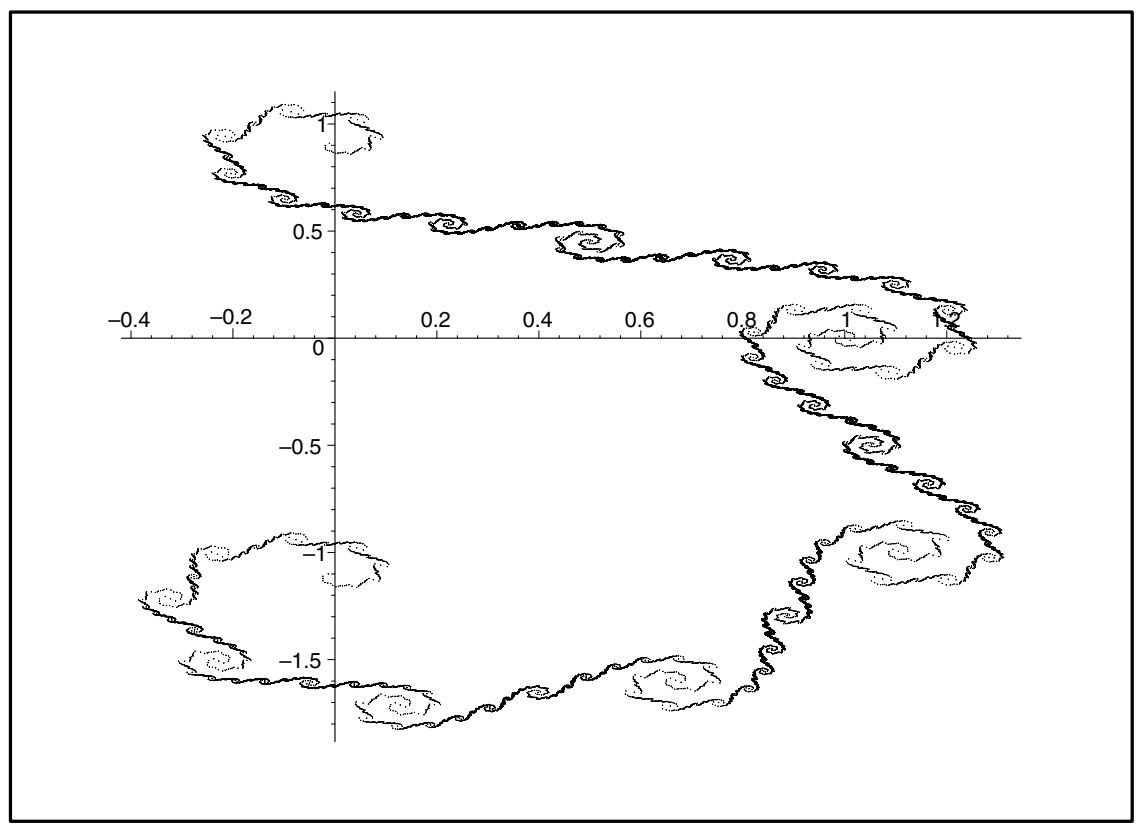

Figure $3 . \mathscr{I}_{\mathrm{p}}, \mathrm{p}=1+0.9 i$. This is a continuous curve! 


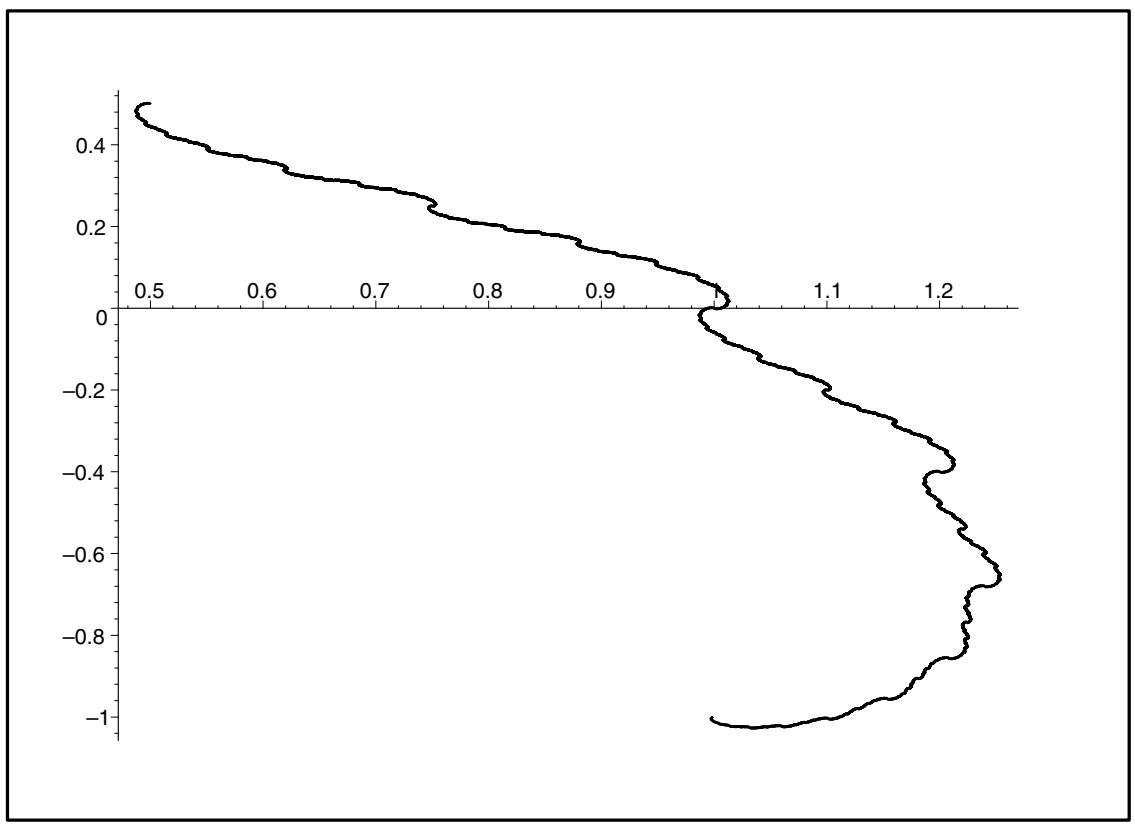

FiguRE $4 . \mathscr{I}_{\mathrm{p}}, \mathrm{p}=1.5+0.5 i$

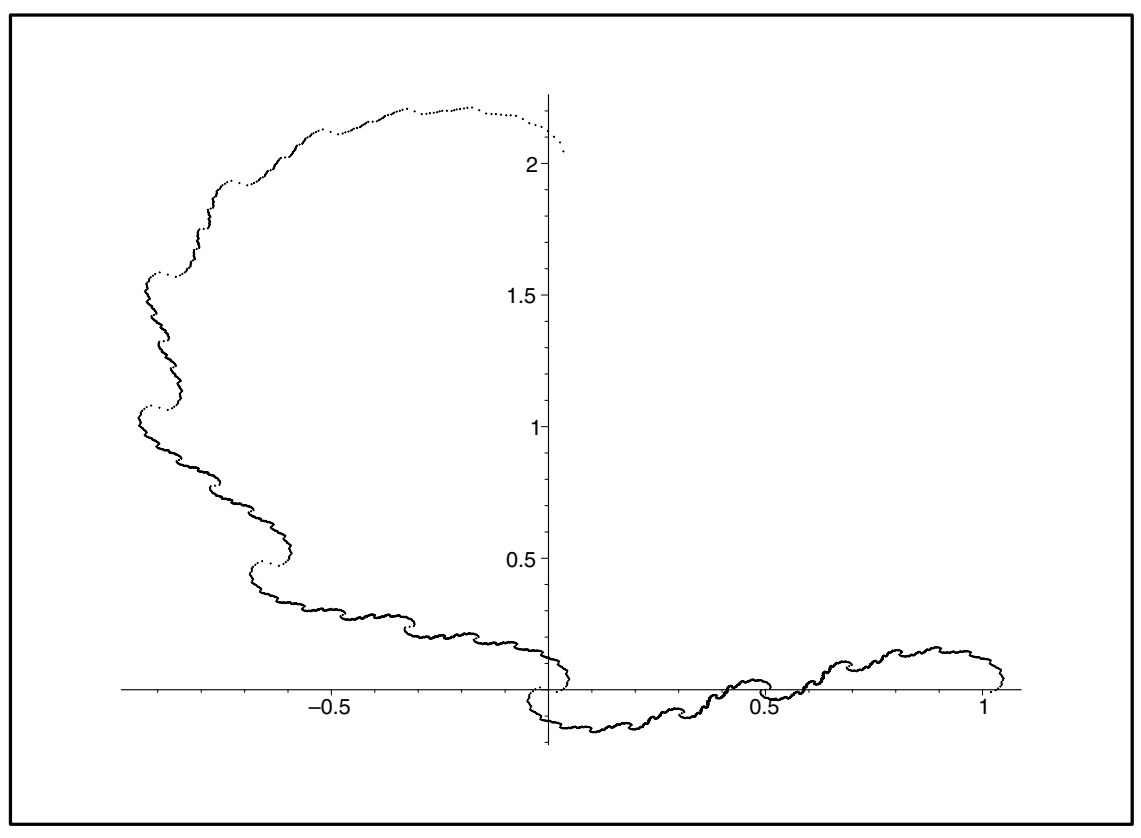

FiguRE $5 .\left.\frac{\mathrm{d}}{\mathrm{d} \mathrm{p}} \mathfrak{X}(\mathrm{p},[0, \infty])\right|_{\mathrm{p}=\mathrm{p}_{0}}, \mathrm{p}_{0}=1.5+0.5 i$ 
Proof of Theorem 3.1. We need the following two results.

Theorem 3.2 (34, p. 57). Let $v_{\nu}, \nu \in \mathbb{N}$ be positive numbers such that

$$
v_{1}<1, \quad v_{\nu}+v_{\nu+1} \leq 1, \text { for } \nu \geq 1
$$

Suppose we are given complex numbers $e_{\nu}, \nu \geq 2$, such that

$$
\left|e_{\nu+1}\right|-\Re\left(e_{\nu+1}\right) \leq v_{\nu}, \quad \nu \geq 1 .
$$

Define the sequence $b_{\nu}$ by the recurrence $b_{1}=1, e_{\nu+1}=\frac{1}{b_{\nu} b_{\nu+1}}, \nu \geq 1$. Then the continued fraction

$$
\mathcal{F}=\frac{1}{1+\frac{e_{2}}{1+\frac{e_{3}}{1+\frac{e_{4}}{\ddots}}}}
$$

converges if, and only if, (a) some $e_{\nu}$ vanishes, or $(b) e_{\nu} \neq 0$ for $\nu \geq 2$ and the series $\sum_{\nu=1}^{\infty}\left|b_{\nu}\right|$ diverges. Moreover, if $e_{\nu}(z): \mathbf{K}_{1} \rightarrow \mathbf{K}_{2}$ are analytic functions of a complex variable, $\mathbf{K}_{1}$ and $\mathbf{K}_{2}$ are compact sets, (11) and (12) are satisfied, and the above series diverges uniformly, then the continued fraction converges uniformly for all $z \in \mathbf{K}_{1}$.

Theorem 3.3 ([34, p. 60). If all $v_{\nu}=\frac{1}{2}$, and the conditions (a) and (b) of Theorem 3.2 hold, then $|\mathcal{F}-1| \leq 1, \mathcal{F} \neq 0$.

For $a, b \in \mathbb{N}, \mathrm{p} \in \mathbb{C},|\mathrm{p}-2| \leq 1$, define the rational functions

$$
\begin{aligned}
W_{a}(\mathrm{p}) & =\frac{\mathrm{p}^{a}-1}{\mathrm{p}^{a+1}-\mathrm{p}^{a}} \\
T_{a, b}(\mathrm{p}) & =W_{a}^{-1}(\mathrm{p}) W_{b}^{-1}(\mathrm{p}) \mathrm{p}^{-a}=\frac{(\mathrm{p}-1)^{2} \mathrm{p}^{b}}{\left(\mathrm{p}^{a}-1\right)\left(\mathrm{p}^{b}-1\right)}, \quad T_{a, \infty}(\mathrm{p})=\frac{(\mathrm{p}-1)^{2}}{\left(\mathrm{p}^{a}-1\right)}
\end{aligned}
$$

Since, for fixed $\mathrm{p} \neq 1, W_{a}(\mathrm{p}) \rightarrow \mathrm{p}-1$, as $a \rightarrow \infty$, then there exist two constants $k_{1}=k_{1}(\mathrm{p})$ and $k_{2}=k_{2}(\mathrm{p})$, such that

$$
0<k_{1} \leq\left|W_{a}(\mathrm{p})\right| \leq k_{2}<+\infty, \quad a \in \mathbb{N} .
$$

Let $x \geq 1, x=\left[a_{1}, a_{2}, a_{3}, \ldots\right]$, be an irrational number, $a_{i} \in \mathbb{N}$. Let us consider the continued fraction

$$
\mathcal{F}(\mathrm{p}, x)=\mathcal{F}\left(\mathrm{p}, a_{1}, a_{2}, \ldots\right)=\frac{1}{1+\frac{T_{a_{1}, a_{2}}(\mathrm{p})}{1+\frac{T_{a_{2}, a_{3}}(\mathrm{p})}{1+\frac{T_{a_{3}, a_{4}}(\mathrm{p})}{\ddots}}}} .
$$


If $x=\left[a_{1}, a_{2}, \ldots, a_{\kappa}\right] \geq 1$ is rational, let us define

$$
\mathcal{F}(\mathrm{p}, x)=\mathcal{F}\left(\mathrm{p}, a_{1}, a_{2}, \ldots, a_{\kappa}\right)=\frac{1}{1+\frac{T_{a_{1}, a_{2}}(\mathrm{p})}{1+\frac{T_{a_{2}, a_{3}}(\mathrm{p})}{1+\frac{\ddots}{1+T_{a_{\kappa}, \infty}}}}} .
$$

From the definition, this continued fraction obeys the following rule:

$$
\mathcal{F}\left(\mathrm{p}, a_{1}, a_{2}, \ldots\right)=\frac{1}{1+T_{a_{1}, a_{2}}(\mathrm{p}) \cdot \mathcal{F}\left(\mathrm{p}, a_{2}, a_{3} \ldots\right)} .
$$

We will now apply Theorem 3.2 to $\mathcal{F}\left(\mathrm{p}, a_{1}, a_{2}, a_{3}, \ldots\right)$. Suppose $x$ is irrational. Thus, let $e_{\nu}=T_{a_{\nu-1}, a_{\nu}}(\mathrm{p}), \nu \geq 2$. Let us define constants

$$
\mu(a, b)=\sup _{\mathrm{p} \in \mathbb{C},|\mathrm{p}-2| \leq 1}\left|T_{a, b}(\mathrm{p})\right|-\Re\left(T_{a, b}(\mathrm{p})\right) .
$$

By Lemma A.1. $\mu(a, b)+\mu(b, c)<0.76, a, b, c \in \mathbb{N}$. Further, from the definition in Theorem 3.2 it follows that

$$
\begin{aligned}
b_{2 \nu} & =W_{a_{1}}(\mathrm{p}) W_{a_{2 \nu}}(\mathrm{p}) \mathrm{p}^{a_{2 \nu-1}-\ldots+a_{3}-a_{2}+a_{1}}, \\
b_{2 \nu+1} & =W_{a_{1}}^{-1}(\mathrm{p}) W_{a_{2 \nu+1}}(\mathrm{p}) \mathrm{p}^{a_{2 \nu}-\ldots-a_{3}+a_{2}-a_{1}} .
\end{aligned}
$$

It is obvious that the series $\sum_{\nu=1}^{\infty}\left|b_{\nu}\right|$ diverges. Hence, Theorem 3.2 tells us that the continued fraction converges, and that for fixed irrational $x=\left[a_{1}, a_{2}, \ldots\right]>1$, $\mathcal{F}\left(\mathrm{p}_{0}, a_{1}, a_{2}, \ldots\right)$ is an analytic function in $\mathrm{p}_{0}$ in some small neighborhood of $\mathrm{p}$. For rational $x$ this is in fact a rational function.

As is shown in [34, the $\nu$ th convergent of the continued fraction (13) (denote it by $\frac{A_{\nu}}{B_{\nu}}$ ) is equal to the $\nu$ th convergent (denote it by $\frac{P_{\nu}}{Q_{\nu}}$ ) of the continued fraction

$$
\frac{1}{b_{1}+\frac{1}{b_{2}+\frac{1}{b_{3}+\frac{1}{\ddots}}} .}
$$

Moreover, since (11) and (12) are satisfied, we have that, for a certain positive constant $k=k\left(b_{1}, b_{2}, b_{3}\right)(34$, pp. 55-56),

$$
\begin{aligned}
\left|Q_{2 \nu}\right| & \geq k\left(1+\left|b_{2}\right|+\left|b_{4}\right|+\ldots+\left|b_{2 \nu}\right|\right), \\
\left|Q_{2 \nu+1}\right| & \geq k\left(1+\left|b_{3}\right|+\left|b_{5}\right|+\ldots+\left|b_{2 \nu+1}\right|\right), \\
\left|\frac{A_{\nu+1}}{B_{\nu+1}}-\frac{A_{\nu}}{B_{\nu}}\right| & =\frac{1}{\left|Q_{\nu} Q_{\nu+1}\right|} .
\end{aligned}
$$

Now we have

Proposition 3.4. Fix $\mathrm{p} \in \mathbb{C},|\mathrm{p}-2| \leq 1, \mathrm{p} \neq 1$. Let $x=\left[a_{1}, a_{2}, \ldots\right] \geq 1$ be a real number. The function $\mathcal{F}(\mathrm{p}, x):[1, \infty] \rightarrow \mathbb{C}$ is continuous.

Proof. Fix an irrational $x>1$. Let $\delta>0$ and $y \geq 1$ be such that $|x-y|<\delta$. Then there exists an $N$ such that the first $N$ partial quotients of $x$ and $y$ coincide, 
$N=N(\delta) \rightarrow \infty$ as $\delta \rightarrow 0$. Consequently, let the corresponding convergents to $\mathcal{F}(\mathrm{p}, x)$ and $\mathcal{F}(\mathrm{p}, y)$ be, respectively,

$$
\begin{array}{lllll}
\frac{A_{1}}{B_{1}}, & \frac{A_{2}}{B_{2}}, \quad \ldots, \frac{A_{N}}{B_{N}}, & \frac{A_{N+1}}{B_{N+1}}, & \frac{A_{N+2}}{B_{N+2}}, \ldots ; \\
\frac{A_{1}}{B_{1}}, & \frac{A_{2}}{B_{2}}, & \ldots, \frac{A_{N}}{B_{N}}, & \frac{A_{N+1}^{\prime}}{B_{N+1}^{\prime}}, & \frac{A_{N+2}^{\prime}}{B_{N+2}^{\prime}} \ldots
\end{array}
$$

Now, combining (14), (16) and (17) we see that

$$
\begin{aligned}
\left|Q_{2 \nu} Q_{2 \nu+1}\right|>k^{2} k_{1}^{3} k_{2}^{-1} & \times\left(|\mathrm{p}|^{a_{1}}+|\mathrm{p}|^{a_{3}-a_{2}+a_{1}}+\ldots+|\mathrm{p}|^{a_{2 \nu-1}-\ldots+a_{3}-a_{2}+a_{1}}\right) \\
& \times\left(|\mathrm{p}|^{a_{2}-a_{1}}+|\mathrm{p}|^{a_{4}-a_{3}+a_{2}-a_{1}}+\ldots+|\mathrm{p}|^{a_{2 \nu}-\ldots-a_{3}+a_{2}-a_{1}}\right) .
\end{aligned}
$$

Denote $c_{1}=k^{2} k_{1}^{3} k_{2}^{-1}$. Let $|\mathrm{p}|^{a_{2 \ell-1}-\ldots+a_{3}-a_{2}+a_{1}}=\lambda_{\ell}, 1 \leq \ell \leq \nu$. The above inequality and the arithmetic-harmonic mean inequality give

$$
\begin{aligned}
\left|Q_{2 \nu} Q_{2 \nu+1}\right| & >c_{1}\left(\lambda_{1}+\lambda_{2}+\ldots+\lambda_{\nu}\right) \cdot\left(|\mathrm{p}|^{a_{2}} \lambda_{1}^{-1}+|\mathrm{p}|^{a_{4}} \lambda_{2}^{-1}+\ldots+|\mathrm{p}|^{a_{2 \nu}} \lambda_{\nu}^{-1}\right) \\
& \geq|\mathrm{p}| c_{1}\left(\lambda_{1}+\lambda_{2}+\ldots+\lambda_{\nu}\right) \cdot\left(\lambda_{1}^{-1}+\lambda_{2}^{-1}+\ldots+\lambda_{\nu}^{-1}\right) \geq|\mathrm{p}| c_{1} \nu^{2}, \quad \nu \geq 1 .
\end{aligned}
$$

Analogously we prove that $\left|Q_{2 \nu-1} Q_{2 \nu}\right|>|\mathrm{p}| c_{2} \nu^{2}, \nu \geq 2$. Thus, $\left|Q_{\nu} Q_{\nu+1}\right|>c \nu^{2}$ for a certain real $c>0, \nu \geq 2$. We see that (17) yields

$$
\left|\mathcal{F}(\mathrm{p}, x)-\frac{A_{N}}{B_{N}}\right|<\sum_{\nu=N}^{\infty} \frac{1}{\left|Q_{\nu} Q_{\nu+1}\right|} \leq \sum_{\nu=N}^{\infty} \frac{c^{-1}}{\nu^{2}}<\frac{c^{-1}}{N-1} ;\left|\mathcal{F}(\mathrm{p}, y)-\frac{A_{N}}{B_{N}}\right|<\frac{c^{-1}}{N-1} .
$$

This implies that $|\mathcal{F}(\mathrm{p}, x)-\mathcal{F}(\mathrm{p}, y)|<\frac{2 c^{-1}}{N-1}$. In case $x$ is rational we argue in a similar way. In this case note that real numbers close to $x=\left[a_{1}, a_{2}, \ldots, a_{\kappa}\right]$ are of the form either $\left[a_{1}, a_{2}, \ldots, a_{\kappa}, T, \ldots\right]$ or $\left[a_{1}, a_{2}, \ldots, a_{\kappa}-1,1, T, \ldots\right]$ for $T$ sufficiently large. The case $x=\infty$ is analogous. This establishes the validity of the proposition.

Eventually, for real numbers $x \geq 0, x=\left[a_{0}, a_{1}, a_{2}, \ldots\right]$, let us define

$$
\mathfrak{X}\left(\mathrm{p},\left[a_{0}, a_{1}, \ldots\right]\right)=W_{a_{0}}(\mathrm{p})+\frac{\mathrm{p}^{-a_{0}}}{W_{a_{1}}(\mathrm{p})+\frac{\mathrm{p}^{-a_{1}}}{W_{a_{2}}(\mathrm{p})+\frac{\mathrm{p}^{-a_{2}}}{W_{a_{3}}(\mathrm{p})+\ddots}} .} .
$$

After an equivalence transformation ([34, p.19), this can be given an expression

$$
\mathfrak{X}\left(\mathrm{p},\left[a_{0}, a_{1}, \ldots\right]\right)=W_{a_{0}}(\mathrm{p})+\mathrm{p}^{-a_{0}} W_{a_{1}}^{-1}(\mathrm{p}) \cdot \mathcal{F}\left(\mathrm{p}, a_{1}, a_{2}, a_{3}, \ldots\right) .
$$

From the very construction, this function satisfies the functional equations (9), is continuous at $x=1$ and thus, according to Proposition 3.4, is continuous for $x \in[0, \infty]$. Obviously, (9) determines the values of $\mathfrak{X}(\mathrm{p}, x)$ at rational $x$ uniquely; hence a continuous solution to (9) is unique. We are left to show that the image of the curve $\mathscr{I}_{\mathrm{p}}$ is contained outside the circle $|z+1| \leq \frac{3}{4}$. This is equivalent to the statement that $\frac{\mathrm{p} \mathscr{I}_{\mathrm{p}}}{\mathscr{I}_{\mathrm{p}}+1}$ is contained inside the circle $|z-\mathrm{p}| \leq \frac{4 \mathrm{p}}{3}$. But the points on $\frac{\mathrm{p} \mathscr{I}_{\mathrm{p}}}{\mathscr{I}_{\mathrm{p}}+1}$ are exactly the point on the curve $\mathscr{I}_{\mathrm{p}}$ with $a_{0}=0$. Thus, we need to show that

$$
\left|\mathrm{p}^{-1} \mathfrak{X}\left(\mathrm{p},\left[0, a_{1}, a_{2}, \ldots\right]\right)-1\right|=\left|\mathrm{p}^{-1} W_{a_{1}}^{-1} \mathcal{F}\left(\mathrm{p}, a_{1}, a_{2}, \ldots\right)-1\right| \leq \frac{4}{3} .
$$


Unfortunately, we cannot apply Theorem 3.3 directly to all $\mathrm{p},|\mathrm{p}-2| \leq 1$, since the table above Lemma A.1 shows that $\mu(1, b)>\frac{1}{2}$ for infinitely many $b$. The maximum values $\mu(1, b)$ (see the definition of this constant) are produced by points $\mathrm{p}$ close either to $\chi=2+e^{2 \pi i / 3}$, or to $\bar{\chi}$. For this reason let us introduce

$$
\mu^{\star}(a, b)=\sup _{\mathrm{p} \in \mathbb{C},|\mathrm{p}-2| \leq 1,|\mathrm{p}-\chi| \geq 0.19,|\mathrm{p}-\bar{\chi}| \geq 0.19}\left|T_{a, b}(\mathrm{p})\right|-\Re\left(T_{a, b}(\mathrm{p})\right) .
$$

Then indeed $\mu^{\star}(a, b)<\frac{1}{2}$ for all $a, b \in \mathbb{N}$. Thus, Theorem 3.3 gives $\mid \mathcal{F}\left(\mathrm{p}, a_{1}, a_{2}, \ldots\right)-$ $1 \mid \leq 1$, and the statement (19) follows from Lemma A.3. In case $|p-2| \leq 1$, $|\mathrm{p}-\chi|<0.19$ (or $|\mathrm{p}-\bar{\chi}|<0.19$ ) we use another theorem by Wall ([34], p. 152), which describes the value region of a continued fraction (13), provided elements $e_{\nu}$ belong to the compact domain in the parabolic region $|z|-\Re\left(z e^{i \phi}\right) \leq 2 h \cos ^{2} \frac{\phi}{2}$, for certain fixed $-\pi<\phi<+\pi, 0<h \leq \frac{1}{4}$. We omit the details. This proves part (ii). In a similar fashion we prove part (iii). Finally, a direct inspection shows that slightly modified proofs remain valid in case $p=1$ if we define a function to be analytic at $p=1$, if it possesses all higher $p$-derivatives, while remaining inside the disc $|p-2| \leq 1$.

Definition 3.5. We define the Minkowski p-question mark function $F_{\mathrm{p}}(x): \mathscr{I}_{\mathrm{p}} \rightarrow$ $[0,1]$, by

$$
F_{\mathrm{p}}(\mathfrak{X}(\mathrm{p}, x))=F(x), \quad x \in[0, \infty] .
$$

\section{Properties of integral transforms of $F_{\mathrm{p}}(x)$}

For given $\mathrm{p},|\mathrm{p}-2| \leq 1$, we define

$$
\chi_{n}=\frac{\mathrm{p}+\mathrm{p}^{n-1}-2}{\mathrm{p}^{n-1}(\mathrm{p}-1)}, \quad \mathscr{I}_{n}=\left[\chi_{n}, \chi_{n+1}\right]=\mathfrak{X}(\mathrm{p},[n, n+1]) \text { for } n \in \mathbb{N}_{0} .
$$

Complex numbers $\chi_{n}$ stand for the analogue of nonnegative integers on the curve $\mathscr{I}_{\mathrm{p}}$. In other words, $\chi_{n}=\mathcal{U}^{n}(\mathrm{p}-1)$. We consider $\mathscr{I}_{n}$ as part of the curve $\mathscr{I}_{\mathrm{p}}$ contained between the points $\chi_{n}$ and $\chi_{n+1}$. Thus, $\chi_{0}=\mathrm{p}-1, \chi_{1}=1$, and the sequence $\chi_{n}$ is "increasing", in the sense that $\chi_{j}$ as a point on a curve $\mathscr{I}_{\mathrm{p}}$ is between $\chi_{i}$ and $\chi_{k}$ if $i<j<k$. Moreover, $\bigcup_{n=0}^{\infty} \mathscr{I}_{n} \cup\left\{\frac{1}{\mathrm{p}-1}\right\}=\mathscr{I}_{\mathrm{p}}$.

Proposition 4.1. Let $\omega(x): \mathscr{I}_{\mathrm{p}} \rightarrow \mathbb{C}$ be a continuous function. Then

$$
\int_{\mathscr{I}_{\mathrm{p}}} \omega(x) \mathrm{d} F_{\mathrm{p}}(x)=\sum_{n=0}^{\infty} \frac{1}{2^{n+1}} \int_{\mathscr{I}_{\mathrm{p}}} \omega\left(\frac{x}{\mathrm{p}^{n-1}(x+1)}+\frac{\mathrm{p}^{n}-1}{\mathrm{p}^{n+1}-\mathrm{p}^{n}}\right) \mathrm{d} F_{\mathrm{p}}(x) .
$$

Proof. Indeed, using (10) we obtain

$$
\begin{array}{r}
\int_{\mathscr{I}_{\mathrm{p}}} \omega(x) \mathrm{d} F_{\mathrm{p}}(x)=\sum_{n=0}^{\infty} \int_{\mathscr{I}_{n}} \omega(x) \mathrm{d} F_{\mathrm{p}}(x)=\sum_{n=0}^{\infty} \int_{\mathcal{T}^{n}\left(\mathscr{I}_{0}\right)} \omega(x) \mathrm{d} F_{\mathrm{p}}(x) \\
\stackrel{x \rightarrow \mathcal{T}^{n}}{=} \sum_{n=0}^{\infty} \frac{1}{2^{n}} \int_{\mathscr{I}_{0}} \omega\left(\mathcal{T}^{n} x\right) \mathrm{d} F_{\mathrm{p}}(x) \stackrel{x \rightarrow \mathcal{U}}{=} \sum_{n=0}^{\infty} \frac{1}{2^{n+1}} \int_{\mathscr{I}_{\mathrm{p}}} \omega\left(\mathcal{T}^{n} \mathcal{U} x\right) \mathrm{d} F_{\mathrm{p}}(x),
\end{array}
$$

and this is exactly the statement of the proposition. 
For $L, T \in \mathbb{N}_{0}$, let us introduce

$$
B_{L, T}(\mathrm{p})=\sum_{n=0}^{\infty} \frac{1}{2^{n+1} \mathrm{p}^{T n}}\left(\frac{\mathrm{p}^{n}-1}{\mathrm{p}^{n+1}-\mathrm{p}^{n}}\right)^{L} .
$$

For example,

$$
\begin{aligned}
B_{0, T} & =\frac{\mathrm{p}^{T}}{2 \mathrm{p}^{T}-1}, \quad B_{1, T}(\mathrm{p})=\frac{\mathrm{p}^{T}}{\left(2 \mathrm{p}^{T}-1\right)\left(2 \mathrm{p}^{T+1}-1\right)}, \\
B_{2, T}(\mathrm{p}) & =\frac{\mathrm{p}^{T}\left(2 \mathrm{p}^{T+1}+1\right)}{\left(2 \mathrm{p}^{T+2}-1\right)\left(2 \mathrm{p}^{T+1}-1\right)\left(2 \mathrm{p}^{T}-1\right)}, \\
B_{3, T}(\mathrm{p}) & =\frac{\mathrm{p}^{T}\left(4 \mathrm{p}^{2 T+3}+4 \mathrm{p}^{T+2}+4 \mathrm{p}^{T+1}+1\right)}{\left(2 \mathrm{p}^{T+3}-1\right)\left(2 \mathrm{p}^{T+2}-1\right)\left(2 \mathrm{p}^{T+1}-1\right)\left(2 \mathrm{p}^{T}-1\right)}, \\
B_{4, T}(\mathrm{p}) & =\frac{\mathrm{p}^{T}\left(2 \mathrm{p}^{T+2}+1\right)\left(4 \mathrm{p}^{2 T+4}+6 \mathrm{p}^{T+3}+8 \mathrm{p}^{T+2}+6 \mathrm{p}^{T+1}+1\right)}{\left(2 \mathrm{p}^{T+4}-1\right)\left(2 \mathrm{p}^{T+3}-1\right)\left(2 \mathrm{p}^{T+2}-1\right)\left(2 \mathrm{p}^{T+1}-1\right)\left(2 \mathrm{p}^{T}-1\right)} .
\end{aligned}
$$

As is easy to see, $B_{L, T}(\mathrm{p})$ are rational functions in $\mathrm{p}$ for $L, T \in \mathbb{N}_{0}$. Indeed,

$$
\begin{aligned}
B_{L, T}(\mathrm{p}) & =\frac{1}{(\mathrm{p}-1)^{L}} \cdot \sum_{n=0}^{\infty} \frac{1}{\mathrm{p}^{T n} 2^{n+1}}\left(1-\frac{1}{\mathrm{p}^{n}}\right)^{L} \\
& =\frac{1}{2(\mathrm{p}-1)^{L}} \cdot \sum_{s=0}^{L}(-1)^{s}\left(\begin{array}{c}
L \\
s
\end{array}\right) \sum_{n=0}^{\infty} \frac{1}{2^{n} \mathrm{p}^{n(s+T)}} \\
& =\frac{\mathrm{p}^{T}}{(\mathrm{p}-1)^{L}} \cdot \sum_{s=0}^{L}(-1)^{s}\left(\begin{array}{c}
L \\
s
\end{array}\right) \frac{\mathrm{p}^{s}}{2 \mathrm{p}^{s+T}-1} \\
& =\frac{\mathrm{p}^{T} \mathscr{R}_{L, T}(\mathrm{p})}{\left(2 \mathrm{p}^{T+L}-1\right)\left(2 \mathrm{p}^{T+L-1}-1\right) \cdot \ldots \cdot\left(2 \mathrm{p}^{T+1}-1\right)\left(2 \mathrm{p}^{T}-1\right)}
\end{aligned}
$$

where $\mathscr{R}_{L, T}(\mathrm{p})$ are polynomials. This follows from the observation that $\mathrm{p}=1$ is a root of the numerator of multiplicity not less than $L$.

As in the case $\mathrm{p}=1$, our main concerns are the moments of the distributions $F_{\mathrm{p}}(x)$, which for $L \in \mathbb{N}$ are defined by

$$
\begin{aligned}
m_{L}(\mathrm{p}) & =2 \int_{\mathscr{I}_{0}} x^{L} \mathrm{~d} F_{\mathrm{p}}(x)=\int_{\mathscr{I}_{\mathrm{p}}}\left(\frac{\mathrm{p} x}{x+1}\right)^{L} \mathrm{~d} F_{\mathrm{p}}(x) \\
& =2 \int_{0}^{1} \mathfrak{X}^{L}(\mathrm{p}, x) \mathrm{d} F(x)=\lim _{n \rightarrow \infty} 2^{2-n} \sum_{a_{1}+a_{2}+\ldots+a_{s}=n}\left[0, a_{1}, a_{2}, . ., a_{s}\right]_{\mathrm{p}}^{L} \\
M_{L}(\mathrm{p}) & =\int_{\mathscr{I}_{\mathrm{p}}} x^{L} \mathrm{~d} F_{\mathrm{p}}(x) .
\end{aligned}
$$

Thus, if $\sup _{z \in \mathscr{I}_{\mathrm{p}}}|z|=\rho_{\mathrm{p}}>1$, which is finite for $\Re \mathrm{p} \geq 1, \mathrm{p} \neq 1$ (see Section 3), then $M_{L}(\mathrm{p}) \leq \rho_{\mathrm{p}}^{L}$.

Proposition 4.2. The function $m_{L}(\mathrm{p})$ is analytic in the disc $|\mathrm{p}-2| \leq 1$, including its boundary. In particular, if in this disc,

$$
\widehat{m}_{L}(\mathrm{p}):=\frac{m_{L}(\mathrm{p})}{\mathrm{p}^{L}}=\sum_{v=0}^{\infty} \eta_{v, L}(\mathrm{p}-2)^{v}
$$

then for any $M \in \mathbb{N}$, one has the estimate $\eta_{v, L} \ll v^{-M}$ as $v \rightarrow \infty$. 
Proof. The function $\mathfrak{X}(\mathrm{p}, x)$ possesses a derivative in $\mathrm{p}$ for $\Re \mathrm{p} \geq 1,|\mathrm{p}-2| \leq 1$, and these are bounded and continuous functions for $x \in \mathbb{R}_{+}$. Therefore $m_{L}(\mathrm{p})$ has a derivative. For $\mathrm{p}=1, M \in \mathbb{M}$ there exists $\frac{\partial^{M}}{\partial \mathrm{p}^{M}} \mathfrak{X}(\mathrm{p}, x) \ll x^{M+1}$, and it is a continuous function for irrational $x$. Additionally, $F^{\prime}(x)=0$ for $x \in \mathbb{Q}_{+}$. This proves the analyticity of $m_{L}(p)$ in the disc $|p-2| \leq 1$. Then an estimate for the Taylor coefficients is the standard fact from Fourier analysis. In fact,

$$
\eta_{v, L}=\int_{0}^{1} \widehat{m}_{L}\left(2+e^{2 \pi i \vartheta}\right) e^{-2 \pi i v \vartheta} \mathrm{d} \vartheta .
$$

The function $\widehat{m}_{L}\left(2+e^{2 \pi i \vartheta}\right) \in \mathrm{C}^{\infty}(\mathbb{R})$; hence the iteration of integration by parts implies the needed estimate.

Proposition 4.3. The functions $M_{L}(\mathrm{p})$ and $m_{L}(\mathrm{p})$ are related via rational functions $B_{L, T}(\mathrm{p})$ in the following way:

$$
M_{L}(\mathrm{p})=\sum_{s=0}^{L} m_{s}(\mathrm{p}) B_{L-s, s}(\mathrm{p})\left(\begin{array}{c}
L \\
s
\end{array}\right) .
$$

Proof. Indeed, this follows from the definitions and Proposition 4.1 in the case $\omega(x)=x^{L}$.

Let us introduce, following [1] in the case $p=1$, the following generating functions:

$$
\begin{aligned}
\mathfrak{m}_{\mathrm{p}}(t) & =\sum_{L=0}^{\infty} m_{L}(\mathrm{p}) \frac{t^{L}}{L !}=2 \int_{\mathscr{I}_{0}} e^{x t} \mathrm{~d} F_{\mathrm{p}}(x)=\int_{\mathscr{I}_{\mathrm{p}}} \exp \left(\frac{\mathrm{p} x t}{x+1}\right) \mathrm{d} F_{\mathrm{p}}(x) ; \\
G_{\mathrm{p}}(z) & =\sum_{L=1}^{\infty} \frac{m_{L}(\mathrm{p})}{\mathrm{p}^{L}} z^{L-1}=\int_{\mathscr{I}_{\mathrm{p}}} \frac{1}{x+1-z} \mathrm{~d} F_{\mathrm{p}}(x)=\int_{0}^{\infty} \frac{1}{\mathfrak{X}(\mathrm{p}, x)+1-z} \mathrm{~d} F(x) .
\end{aligned}
$$

The situation $\mathrm{p}=2$ is particularly important, since all these functions can be explicitly calculated, and it provides the case where all the subsequent results can be checked directly and the starting point in proving Theorem 1.2 Thus,

$$
\mathfrak{m}_{2}(t)=e^{t}, \quad G_{2}(z)=\frac{1}{2-z} .
$$

By the definition, the expressions $m_{L}(\mathrm{p}) / \mathrm{p}^{L}$ are Taylor coefficients of $G_{\mathrm{p}}(z)$ at $z=0$. Differentiation $L-1$ times under the integral defining $G_{\mathrm{p}}(z)$ and substitution $z=1$ gives

$$
G_{\mathrm{p}}^{(L-1)}(1)=(L-1) ! \int_{\mathscr{I}_{\mathrm{p}}} \frac{1}{x^{L}} \mathrm{~d} F_{\mathrm{p}}(x)=(L-1) ! M_{L}(\mathrm{p}) \Rightarrow G_{\mathrm{p}}(z+1)=\sum_{L=0}^{\infty} M_{L}(\mathrm{p}) z^{L-1},
$$

with a radius of convergence equal to $\rho_{\mathrm{p}}^{-1}$. As was proved in [1] and mentioned before, in case $\mathrm{p}=1\left(\rho_{1}=\infty\right)$ this must be interpreted that all derivatives exist at $z=1$. The next proposition shows how the symmetry property reflects in $\mathfrak{m}_{\mathrm{p}}(t)$.

Proposition 4.4. One has

$$
\mathfrak{m}_{\mathbf{p}}(t)=e^{\mathrm{pt}} \mathfrak{m}_{\mathbf{p}}(-t) .
$$


Proof. Indeed,

$$
\begin{aligned}
& \mathfrak{m}_{\mathrm{p}}(t)=\int_{\mathscr{I}_{\mathrm{p}}} \exp \left(\frac{\mathrm{p} x t}{x+1}\right) \mathrm{d} F_{\mathrm{p}}(x)=\int_{\mathscr{I}_{\mathrm{p}}} \exp \left(\mathrm{p} t-\frac{\mathrm{p} t}{x+1}\right) \mathrm{d} F_{\mathrm{p}}(x) \\
& =e^{\mathrm{p} t} \int_{\mathscr{I}_{\mathrm{p}}} \exp \left(-\frac{\mathrm{p} t}{x+1}\right) \mathrm{d} F_{\mathrm{p}}(x) \stackrel{x \rightarrow \frac{1}{x}}{=} e^{\mathrm{p} t} \mathfrak{m}_{\mathrm{p}}(-t) .
\end{aligned}
$$

This result allows us to obtain linear relations among moments $m_{L}(\mathrm{p})$ and the exact value of the first (trivial) moment $m_{1}(\mathrm{p})$.

Corollary 4.5. One has

$$
m_{1}(\mathrm{p})=\frac{\mathrm{p}}{2}, \quad M_{1}(\mathrm{p})=\frac{\mathrm{p}^{2}+2}{4 \mathrm{p}-2} .
$$

Proof. Indeed, the last proposition implies

$$
m_{L}(\mathrm{p})=\sum_{s=0}^{L}\left(\begin{array}{c}
L \\
s
\end{array}\right)(-1)^{s} m_{s}(\mathrm{p}) \mathrm{p}^{L-s}, \quad L \geq 0 .
$$

For $L=1$ this gives the first statement of the corollary. Additionally, Proposition 4.3 for $L=1$ reads as

$$
M_{1}(\mathrm{p})=\frac{\mathrm{p}}{2 \mathrm{p}-1} \cdot m_{1}(\mathrm{p})+\frac{1}{2 \mathrm{p}-1},
$$

and we are done.

\section{Three-term Functional Equation}

Theorem 5.1. The function $G_{\mathrm{p}}(z)$ can be extended to an analytic function in the domain $\mathbb{C} \backslash\left(\mathscr{I}_{\mathrm{p}}+1\right)$. It satisfies the functional equation

$$
\frac{1}{z}+\frac{\mathrm{p}}{z^{2}} G_{\mathrm{p}}\left(\frac{\mathrm{p}}{z}\right)+2 G_{\mathrm{p}}(z+1)=\mathrm{p} G_{\mathrm{p}}(\mathrm{p} z), \text { for } z \notin \frac{\mathscr{I}_{\mathrm{p}}+1}{\mathrm{p}} .
$$

Its consequence is the symmetry property

$$
G_{\mathrm{p}}(z+1)=-\frac{1}{z^{2}} G_{\mathrm{p}}\left(\frac{1}{z}+1\right)-\frac{1}{z} .
$$

Moreover, $G_{\mathrm{p}}(z) \rightarrow 0$ if $\operatorname{dist}\left(z, \mathscr{I}_{\mathrm{p}}\right) \rightarrow \infty$.

Conversely, the function satisfying this functional equation and the regularity property is unique.

Proof. Let $w(x, z)=\frac{1}{x+1-z}$. Then it is straightforward to check that

$$
\begin{aligned}
& w\left(\frac{x+1}{\mathrm{p}}, z+1\right)=\mathrm{p} \cdot w(x, \mathrm{p} z), \\
& w\left(\frac{\mathrm{p}}{x+1}, z+1\right)=-\frac{\mathrm{p}}{z^{2}} w\left(x, \frac{\mathrm{p}}{z}\right)-\frac{1}{z} .
\end{aligned}
$$


Thus, for $|\mathrm{p}-2| \leq 1, \mathrm{p} \neq 2$,

$$
\begin{aligned}
2 G_{\mathrm{p}} & (z+1)=2 \int_{\mathscr{I}_{0}} w(x, z+1) \mathrm{d} F_{\mathrm{p}}(x)+2 \int_{\mathscr{I}_{\mathrm{p}} \backslash \mathscr{I}_{0}} w(x, z+1) \mathrm{d} F_{\mathrm{p}}(x) \\
& =2 \int_{\mathscr{I}_{\mathrm{p}}} w\left(\frac{\mathrm{p} x}{x+1}, z+1\right) \mathrm{d} F_{\mathrm{p}}\left(\frac{\mathrm{p} x}{x+1}\right)+2 \int_{\mathscr{I}_{\mathrm{p}}} w\left(\frac{x+1}{\mathrm{p}}, z+1\right) \mathrm{d} F_{\mathrm{p}}\left(\frac{x+1}{\mathrm{p}}\right) \\
& =\int_{\mathscr{I}_{\mathrm{p}}} w\left(\frac{\mathrm{p}}{x+1}, z+1\right) \mathrm{d} F_{\mathrm{p}}(x)+\int_{\mathscr{I}_{\mathrm{p}}} w\left(\frac{x+1}{\mathrm{p}}, z+1\right) \mathrm{d} F_{\mathrm{p}}(x) \\
& =-\frac{1}{z}-\frac{\mathrm{p}}{z^{2}} G_{\mathrm{p}}\left(\frac{\mathrm{p}}{z}\right)+\mathrm{p} G_{\mathrm{p}}(\mathrm{p} z) .
\end{aligned}
$$

(In the first integral we used a substitution $x \rightarrow \frac{1}{x}$.) The functional equation holds in the case $\mathrm{p}=2$ as well, which can be checked directly. The holomorphicity of $G_{\mathrm{p}}(z)$ follows exactly as in the case $\mathrm{p}=1$ [1]. All we need is the first integral in (20) and the fact that $\mathscr{I}_{\mathrm{p}}$ is a closed set.

As was mentioned, the uniqueness of a function satisfying (22) for $p=1$ was proved in [1. Thus, the converse implication follows from the analytic continuation principle for the function in two complex variables $(\mathrm{p}, z)$. (See Lemma 6.2 below, where the proof in the case $\mathrm{p}=2$ is presented. A similar argument works for general p.)

Corollary 5.2. Let $\mathrm{p} \neq 1$, and let $\mathscr{C}$ be any closed smooth contour which rounds the curve $\mathscr{I}_{\mathrm{p}}+1$ once in the positive direction. Then

$$
\frac{1}{2 \pi i} \oint_{\mathscr{C}} G_{\mathrm{p}}(z) \mathrm{d} z=-1 .
$$

Proof. Indeed, this follows from the functional equation (22), as well as from the symmetry property. It is enough to take a sufficiently large circle $\mathscr{C}=\{|z|=$ $R\}$ such that $\mathscr{C}^{-1}+1$ is contained in a small neighborhood of $z=1$, for which $\left(\mathscr{C}^{-1}+1\right) \cap\left(\mathscr{I}_{\mathrm{p}}+1\right)=\emptyset$. This is possible since $0 \notin \mathscr{I}_{\mathrm{p}}$ (see Theorem 3.1).

We finish by providing an integral equation for $\mathfrak{m}_{\mathrm{p}}(t)$. We indulge in being concise since the argument directly generalizes the one used in [1] to prove the integral functional equation for $\mathfrak{m}(t)$ (in our notation, this is $\mathfrak{m}_{1}(t)$ ).

Proposition 5.3. Let $1 \leq \mathrm{p}<\infty$ be real. Then the function $\mathfrak{m}_{\mathrm{p}}(t)$ satisfies the boundary condition $\mathfrak{m}_{\mathrm{p}}(0)=1$, the regularity property $\mathfrak{m}_{\mathrm{p}}(-t) \ll e^{-\sqrt{t \log 2}}$, and the integral equation

$$
\mathfrak{m}_{\mathrm{p}}(-s)=\int_{0}^{\infty} \mathfrak{m}_{\mathrm{p}}^{\prime}(-t)\left(2 e^{s} J_{0}(2 \sqrt{\mathrm{p} s t})-J_{0}(2 \sqrt{s t})\right) \mathrm{d} t, \quad s \in \mathbb{R}_{+} .
$$

For instance, in the case $\mathrm{p}=1$ this reduces to (4), and in the case $\mathrm{p}=2$ this reads as

$$
2 e^{s} \int_{0}^{\infty} e^{-t} J_{0}(2 \sqrt{2 s t}) \mathrm{d} t=2 e^{s} e^{-2 s}=e^{-s}+e^{-s}=e^{-s}+\int_{0}^{\infty} e^{-t} J_{0}(2 \sqrt{s t}) \mathrm{d} t,
$$

which is an identity [35]. 
Proof. Indeed, the functional equation for $G_{\mathrm{p}}(z)$ in the region $\Re z<-1$ in terms of $\mathfrak{m}_{\mathbf{p}}^{\prime}(t)$ reads as

$$
\frac{1}{z}=\int_{0}^{\infty} \mathfrak{m}_{\mathrm{p}}^{\prime}(-t)\left(\frac{2}{z+1} e^{\frac{\mathrm{p} t}{z+1}}+\frac{1}{z} e^{t z}-\frac{1}{z} e^{\frac{t}{z}}\right) \mathrm{d} t .
$$

Now, multiply this by $e^{-s z}$ and integrate over $\Re z=-\sigma<-1$, where $s>0$ is real. All the remaining steps are exactly the same as in [1].

Remark. If $\mathrm{p} \neq 1$, the regularity bound is easier than in the case $\mathrm{p}=1$. Take, for example, $1<\mathrm{p}<2$. Then

$$
\left|\mathfrak{m}_{\mathrm{p}}(t)\right| \leq \int_{\mathrm{p}-1}^{\frac{1}{\mathrm{p}-1}}\left|\exp \left(\frac{\mathrm{p} x t}{x+1}\right)\right| \mathrm{d} F_{\mathrm{p}}(x)<\int_{\mathrm{p}-1}^{\frac{1}{\mathrm{p}-1}} e^{t} \mathrm{~d} F_{\mathrm{p}}(x)=e^{t} .
$$

Thus, Proposition 4.4 gives $\left|\mathfrak{m}_{\mathrm{p}}(-t)\right|<e^{(1-\mathrm{p}) t}$. The same argument shows that for $\mathrm{p}>2$ we have $\left|\mathfrak{m}_{\mathrm{p}}(-t)\right|<e^{-t}$.

\section{The proof: Approach through $\mathrm{p}=2$}

Let us rewrite the functional equation for $G_{\mathrm{p}}(z)=G(\mathrm{p}, z)$ as

$$
\frac{1}{z}+\frac{\mathrm{p}}{z^{2}} G\left(\mathrm{p}, \frac{\mathrm{p}}{z}\right)+2 G(\mathrm{p}, z+1)=\mathrm{p} G(\mathrm{p}, \mathrm{p} z) .
$$

Direct induction shows that the following "chain-rule" holds:

$$
\begin{array}{r}
\frac{\partial^{n}}{\partial \mathrm{p}^{n}}(\mathrm{p} G(\mathrm{p}, \mathrm{p} z))=\sum_{i+j=n}\left(\begin{array}{c}
n \\
j
\end{array}\right) \mathrm{p} \frac{\partial^{i} \partial^{j}}{\partial \mathrm{p}^{i} \partial z^{j}} G(\mathrm{p}, \mathrm{p} z) z^{j} \\
+\sum_{i+j=n-1} n\left(\begin{array}{c}
n-1 \\
j
\end{array}\right) \frac{\partial^{i} \partial^{j}}{\partial \mathrm{p}^{i} \partial z^{j}} G(\mathrm{p}, \mathrm{p} z) z^{j},
\end{array}
$$

where in the summation it is assumed that $i, j \geq 0$.

Now we will provide rigorous calculations which yield explicit series for $G(\mathrm{p}, z)$ in terms of powers of $(\mathrm{p}-2)$ and certain rational functions. The function $G(\mathrm{p}, z)$ is analytic in $\{|p-2| \leq 1\} \times\left\{|z| \leq \frac{3}{4}\right\}$. This follows from Theorem 3.1 and the integral representation (20). Thus, for $\{|\mathrm{p}-2|<1\} \times\left\{|z| \leq \frac{3}{4}\right\}$ it has a Taylor expansion

$$
G(\mathrm{p}, z)=\sum_{L=1}^{\infty} \sum_{v=0}^{\infty} \eta_{v, L} \cdot z^{L-1}(\mathrm{p}-2)^{v}
$$

Moreover, the function $G\left(2+e^{2 \pi i \vartheta}, \frac{3}{4} e^{2 \pi i \varphi}\right) \in \mathrm{C}^{\infty}(\mathbb{R} \times \mathbb{R})$, and it is double-periodic. Thus,

$\eta_{v, L}=\left(\frac{4}{3}\right)^{L-1} \int_{0}^{1} \int_{0}^{1} G\left(2+e^{2 \pi i \vartheta}, \frac{3}{4} e^{2 \pi i \varphi}\right) e^{-2 \pi i v \vartheta-2 \pi i(L-1) \varphi} \mathrm{d} \vartheta \mathrm{d} \varphi, \quad v \geq 0, \quad L \geq 1$.

A standard trick from Fourier analysis (using iteration of integration by parts) shows that $\eta_{v, L} \ll_{M}(4 / 3)^{L} \cdot(L v)^{-M}$ for any $M \in \mathbb{N}$. Thus, (25) holds for $(\mathrm{p}, z) \in$ $\{|\mathrm{p}-2| \leq 1\} \times\{|z| \leq 3 / 4\}$.

Our idea is a simple one. Indeed, let us look at (20). This implies the Taylor series for $m_{L}(\mathrm{p}) / \mathrm{p}^{L}=\sum_{v=0}^{\infty} \eta_{v, L}(\mathrm{p}-2)^{v}$, convergent in the disc $|\mathrm{p}-2| \leq 1$. Due 
to the absolute convergence, the order of summation in (25) is not essential. This yields

$$
G(\mathrm{p}, z)=\sum_{v=0}^{\infty}(\mathrm{p}-2)^{v}\left(\sum_{L=1}^{\infty} \eta_{v, L} \cdot z^{L-1}\right)
$$

Therefore, let

$$
\left.\frac{1}{n !} \frac{\partial^{n}}{\partial \mathrm{p}^{n}} G(\mathrm{p}, z)\right|_{\mathrm{p}=2}=\mathbf{H}_{n}(z)=\sum_{L=1}^{\infty} \eta_{n, L} \cdot z^{L-1} .
$$

We already know that $\mathbf{H}_{0}(z)=\frac{1}{2-z}$. Though $m_{L}(\mathrm{p})$ are obviously highly transcendental (and mysterious) functions, the series for $\mathbf{H}_{n}(z)$ is in fact a rational function in $z$, and this is the main point of our approach. Moreover, we will show that

$$
\mathbf{H}_{n}(z)=\frac{\mathscr{B}_{n}(z)}{(z-2)^{n+1}},
$$

where $\mathscr{B}_{n}(z)$ is a polynomial with rational coefficients of degree $n-1$ with the reciprocity property $\mathscr{B}_{n}(z+1)=(-1)^{n} z^{n-1} \mathscr{B}_{n}\left(\frac{1}{z}+1\right), \mathscr{B}_{n}(0)=0$. We argue by induction on $n$. First we need an auxiliary lemma.

Let $\mathbb{Q}[z]_{n-1}$ denote the linear space of dimension $n$ of polynomials of degree $\leq$ $n-1$ with rational coefficients. Consider the following linear map $\mathcal{L}_{n-1}: \mathbb{Q}[z]_{n-1} \rightarrow$ $\mathbb{Q}[z]_{n-1}$, defined by

$$
\mathcal{L}_{n-1}(P)(z)=P(z+1)-\frac{1}{2^{n+1}} P(2 z)+\frac{(-1)^{n+1}}{2^{n+1}} P\left(\frac{2}{z}\right) z^{n-1} .
$$

Lemma 6.1. $\operatorname{det}\left(\mathcal{L}_{n-1}\right) \neq 0$. Accordingly, $\mathcal{L}_{n-1}$ is the isomorphism.

Remark. Let $m=\left[\frac{n}{2}\right]$. Then it can be proved that indeed $\operatorname{det}\left(\mathcal{L}_{n-1}\right)=\frac{\prod_{i=1}^{m}\left(4^{i}-1\right)}{2^{m^{2}+m}}$.

Proof. Suppose $P \in \operatorname{ker}\left(\mathcal{L}_{n-1}\right)$. Then a rational function $\mathbf{H}(z)=\frac{P(z)}{(z-2)^{n+1}}$ satisfies the three-term functional equation

$$
\mathbf{H}(z+1)-\mathbf{H}(2 z)+\mathbf{H}\left(\frac{2}{z}\right) \frac{1}{z^{2}}=0, \quad z \neq 1 .
$$

Also, $\mathbf{H}(z)=o(1)$, as $z \rightarrow \infty$. Now the result follows from the next

Lemma 6.2. Let $\Upsilon(z)$ be any analytic function in the domain $\mathbb{C} \backslash\{1\}$. Then if $\mathbf{H}(z)$ is a solution of the equation

$$
\mathbf{H}(z+1)-\mathbf{H}(2 z)+\mathbf{H}\left(\frac{2}{z}\right) \frac{1}{z^{2}}=\Upsilon(z),
$$

such that $\mathbf{H}(z) \rightarrow 0$ as $z \rightarrow \infty, \mathbf{H}(z)$ is analytic in $\mathbb{C} \backslash\{2\}$, then such an $\mathbf{H}(z)$ is unique.

Proof. All we need is to show that with the imposed diminishing condition, homogeneous equation (26) admits only the solution $\mathbf{H}(z) \equiv 0$. Indeed, let $\mathbf{H}(z)$ be such a solution. Put $z \rightarrow 2^{n} z+1$. Thus,

$$
\mathbf{H}\left(2^{n} z+2\right)-\mathbf{H}\left(2^{n+1} z+2\right)+\frac{1}{\left(2^{n} z+1\right)^{2}} \mathbf{H}\left(\frac{2}{2^{n} z+1}\right)=0 .
$$


This is valid for $z \neq 0$ (since $\mathbf{H}(z)$ is allowed to have a singularity at $z=2$ ). Now sum this over $n \geq 0$. Due to the diminishing assumption, one gets (after the additional substitution $z \rightarrow z-2$ )

$$
\mathbf{H}(z)=-\sum_{n=0}^{\infty} \frac{1}{\left(2^{n} z-2^{n+1}+1\right)^{2}} \mathbf{H}\left(\frac{2}{2^{n} z-2^{n+1}+1}\right) .
$$

For clarity, put $z \rightarrow-z$ and consider a function $\widehat{\mathbf{H}}(z)=\mathbf{H}(-z)$. Thus,

$$
\widehat{\mathbf{H}}(z)=-\sum_{n=0}^{\infty} \frac{1}{\left(2^{n} z+2^{n+1}-1\right)^{2}} \widehat{\mathbf{H}}\left(\frac{2}{2^{n} z+2^{n+1}-1}\right) .
$$

Consider this for $z \in[0,2]$. As can be easily seen, then all arguments on the right also belong to this interval. We want to prove the needed result simply by applying the maximum argument. The last identity is still insufficient. For this reason consider its second iteration. This produces a series

$$
\widehat{\mathbf{H}}(z)=\sum_{n, m=0}^{\infty} \frac{1}{\left(2^{n+m+1} z+2^{n+m+2}-2^{n} z-2^{n+1}+1\right)^{2}} \widehat{\mathbf{H}}\left(\omega_{m} \circ \omega_{n}(z)\right),
$$

where $\omega_{n}(z)=\frac{2}{2^{n} z+2^{n+1}-1}$. As we said, $\omega_{m} \circ \omega_{n}(z) \in[0,2]$ for $z \in[0,2]$. Since a function $\widehat{\mathbf{H}}(z)$ is continuous in the interval $[0,2]$, let $z_{0} \in[0,2]$ be such that $M=\left|\widehat{\mathbf{H}}\left(z_{0}\right)\right|=\sup _{z \in[0,2]}|\widehat{\mathbf{H}}(z)|$. Consider the above expression for $z=z_{0}$. Thus,

$$
\begin{aligned}
M & =\left|\widehat{\mathbf{H}}\left(z_{0}\right)\right| \leq \sum_{n, m=0}^{\infty}\left|\frac{1}{\left(2^{n+m+1} z_{0}+2^{n+m+2}-2^{n} z_{0}-2^{n+1}+1\right)^{2}} \widehat{\mathbf{H}}\left(\omega_{m} \circ \omega_{n}\left(z_{0}\right)\right)\right| \\
& \leq M \sum_{n, m=0}^{\infty} \frac{1}{\left(2^{n+m+2}-2^{n+1}+1\right)^{2}}=0.20453_{+} M .
\end{aligned}
$$

This is contradictory unless $M=0$. By the principle of analytic continuation, $\mathbf{H}(z) \equiv 0$, and this proves the lemma.

Remark. Direct inspection of the proof reveals that the statement of the lemma still holds with a weaker assumption that $\mathbf{H}(z)$ is a real-analytic function on $(-\infty, 0]$.

Now, let us differentiate (23) $n$ times with respect to $\mathrm{p}$, use (24) and afterwards substitute $\mathrm{p}=2$. This gives

$$
\begin{aligned}
\sum_{j=1}^{n} \frac{2}{j !} & \frac{\partial^{j}}{\partial z^{j}} \mathbf{H}_{n-j}(2 z) z^{j}+\sum_{j=0}^{n-1} \frac{1}{j !} \frac{\partial^{j}}{\partial z^{j}} \mathbf{H}_{n-j-1}(2 z) z^{j} \\
& -\sum_{j=1}^{n} \frac{2}{j !} \frac{\partial^{j}}{\partial z^{j}} \mathbf{H}_{n-j}\left(\frac{2}{z}\right) \frac{1}{z^{j+2}}-\sum_{j=0}^{n-1} \frac{1}{j !} \frac{\partial^{j}}{\partial z^{j}} \mathbf{H}_{n-j-1}\left(\frac{2}{z}\right) \frac{1}{z^{j+2}} \\
& =2 \mathbf{H}_{n}(z+1)-2 \mathbf{H}_{n}(2 z)+2 \mathbf{H}_{n}\left(\frac{2}{z}\right) \frac{1}{z^{2}} .
\end{aligned}
$$

(We recall here and in the sequel, for example, $\mathbf{H}_{n}^{\prime}(2 z)$ stands for $\left.\frac{\mathrm{d}}{\mathrm{d} u} \mathbf{H}_{n}(u)\right|_{u=2 z}$, and not for $\frac{\mathrm{d}}{\mathrm{d} z}\left(\mathbf{H}_{n}(2 z)\right.$.) We note that this implies the reciprocity property

$$
\mathbf{H}_{n}(z+1)=-\frac{1}{z^{2}} \mathbf{H}_{n}\left(\frac{1}{z}+1\right), \quad n \geq 1 .
$$


A posteriori, this clarifies how the identity $F(x)+F(1 / x)=1$ reflects in the series for $G(z)$, as stated in Theorem 1.2 the reciprocity property (nonhomogeneous for $n=0$ and homogeneous for $n \geq 1$ ) is reflected in each of the summands separately, whereas the three-term functional equation heavily depends on interrelations among $\mathbf{H}_{n}(z)$.

Now, suppose we know all $\mathbf{H}_{j}(z)$ for $j<n$.

Lemma 6.3. The left-hand side of the equation (27) is of the form

$$
\text { l.h.s. }=\frac{\mathscr{J}_{n}(z)}{(z-1)^{n+1}},
$$

where $\mathscr{J}_{n}(z) \in \mathbb{Q}[z]_{n-1}$.

Proof. First, as is clear from the appearance of the l.h.s., we need to verify that $z$ does not divide a denominator, if the l.h.s. is represented as a quotient of two co-prime polynomials. Indeed, using the symmetry property in (23) for the term $G\left(\mathrm{p}, \frac{\mathrm{p}}{z}\right)$, we obtain the three-term functional equation of the form

$$
-\frac{1}{\mathrm{p}-z}-\frac{\mathrm{p}}{(\mathrm{p}-z)^{2}} G\left(\mathrm{p}, \frac{\mathrm{p}}{\mathrm{p}-z}\right)+2 G(\mathrm{p}, z+1)=\mathrm{p} G(\mathrm{p}, \mathrm{p} z) .
$$

Let us perform the same procedure which we followed to arrive at the equation (27). Thus, differentiation $n$ times with respect to $\mathrm{p}$ and substitution of $\mathrm{p}=2$ gives the expression of the form

$$
\text { l.h.s. } 2=2 \mathbf{H}_{n}(z+1)-2 \mathbf{H}_{n}(2 z)-2 \mathbf{H}_{n}\left(\frac{2}{2-z}\right) \frac{1}{(2-z)^{2}},
$$

where lh.s.2 is expressed in terms of $\mathbf{H}_{j}(z)$ for $j<n$. Nevertheless, this time the common denominator of l.h.s. 2 is of the form $(z-1)^{n+1}(z-2)^{n+2}$. As a corollary, $z$ does not divide it. Finally, due to the reciprocity property, for $n \geq 1$ one has

$$
\mathbf{H}_{n}\left(\frac{2}{2-z}\right) \frac{1}{(2-z)^{2}}=-\mathbf{H}_{n}\left(\frac{2}{z}\right) \frac{1}{z^{2}} .
$$

This shows that actually l.h.s. $=$ l.h.s.2, and therefore if this is expressed as a quotient of two polynomials in lowest terms, the denominator is a power of $(z-1)$. Finally, it is obvious that this exponent is exactly $n+1$, and one easily verifies that $\operatorname{deg} \mathscr{J}_{n}(z) \leq n-1$. (Possibly, $\mathscr{J}_{n}(z)$ can be divisible by $(z-1)$, but this does not have an impact on the result.)

Proof of Theorem 1.2. Now, using Lemma6.1, we inherit that there exists a unique polynomial $\mathscr{B}_{n}(z)$ of degree $\leq n-1$ such that $\mathscr{B}_{n}(z)=\frac{1}{2} \mathcal{L}_{n-1}^{-1}\left(\mathscr{J}_{n}\right)(z)$. Summarizing, $\mathbf{H}_{n}(z)=\frac{\mathscr{B}_{n}(z)}{(z-2)^{n+1}}$ solves the equation (27). On the other hand, Lemma 6.2 implies that the solution of (27) we obtained is indeed the unique one. This reasoning proves that for $|\mathrm{p}-2| \leq 1,|z| \leq \frac{3}{4}$ we have the series

$$
G(\mathrm{p}, z)=\sum_{n=0}^{\infty}(\mathrm{p}-2)^{n} \cdot \mathbf{H}_{n}(z) .
$$

This finally establishes the validity of Theorem 1.2 Note also that each summand satisfies the symmetry property. The series converges absolutely for any $z,|z| \leq$ $3 / 4$, and if this holds for $z$, the same does hold for $\frac{z}{z-1}$, which gives the circle $|z+9 / 7| \leq 12 / 7$. 
Curiously, one could formally verify that the function defined by this series does indeed satisfy (22). Indeed, using (27), we get:

$$
\begin{aligned}
& 2 G(\mathrm{p}, z+1)=2 \mathbf{H}_{0}(z+1)+2 \sum_{n=1}^{\infty}(\mathrm{p}-2)^{n} \mathbf{H}_{n}(z+1) \\
&=2 \mathbf{H}_{0}(z+1)+\sum_{n=1}^{\infty}(\mathrm{p}-2)^{n}\left(\sum_{j=0}^{n} \frac{2}{j !} \frac{\partial^{j}}{\partial z^{j}} \mathbf{H}_{n-j}(2 z) z^{j}+\sum_{j=0}^{n-1} \frac{1}{j !} \frac{\partial^{j}}{\partial z^{j}} \mathbf{H}_{n-j-1}(2 z) z^{j}\right. \\
&\left.\quad-\sum_{j=0}^{n} \frac{2}{j !} \frac{\partial^{j}}{\partial z^{j}} \mathbf{H}_{n-j}\left(\frac{2}{z}\right) \frac{1}{z^{j+2}}-\sum_{j=0}^{n-1} \frac{1}{j !} \frac{\partial^{j}}{\partial z^{j}} \mathbf{H}_{n-j-1}\left(\frac{2}{z}\right) \frac{1}{z^{j+2}}\right) .
\end{aligned}
$$

Denote $n-j=s$. Then interchanging the order of summation for the first term of the sum in the brackets, we obtain:

$$
\begin{aligned}
& 2 \sum_{n=1}^{\infty}(\mathrm{p}-2)^{n} \sum_{j=0}^{n} \frac{1}{j !} \frac{\partial^{j}}{\partial z^{j}} \mathbf{H}_{n-j}(2 z) z^{j}=2 \sum_{s=0}^{\infty} \sum_{j=0}^{\infty}(\mathrm{p}-2)^{j+s} \frac{1}{j !} \frac{\partial^{j}}{\partial z^{j}} \mathbf{H}_{s}(2 z) z^{j}-2 \mathbf{H}_{0}(2 z) \\
& \quad=2 \sum_{s=0}^{\infty}(\mathrm{p}-2)^{s} \mathbf{H}_{s}(2 z+(\mathrm{p}-2) z)-2 \mathbf{H}_{0}(2 z)=2 G(\mathrm{p}, \mathrm{p} z)-2 \mathbf{H}_{0}(2 z) .
\end{aligned}
$$

The same works for the second sum:

$$
\sum_{n=1}^{\infty}(\mathrm{p}-2)^{n} \sum_{j=0}^{n-1} \frac{1}{j !} \frac{\partial^{j}}{\partial z^{j}} \mathbf{H}_{n-j-1}(2 z) z^{j}=(\mathrm{p}-2) G(\mathrm{p}, \mathrm{p} z) .
$$

We perform the same interchange of summation for the second and the third summand, respectively. Thus, this yields

$$
\begin{array}{r}
2 G(\mathrm{p}, z+1)=\mathrm{p} G(\mathrm{p}, \mathrm{p} z)-\frac{\mathrm{p}}{z^{2}} G\left(\mathrm{p}, \frac{\mathrm{p}}{z}\right)+2 \mathbf{H}_{0}(z+1)-2 \mathbf{H}_{0}(2 z)+\frac{2}{z^{2}} \mathbf{H}_{0}\left(\frac{2}{z}\right) \\
=\mathrm{p} G(\mathrm{p}, \mathrm{p} z)-\frac{\mathrm{p}}{z^{2}} G\left(\mathrm{p}, \frac{\mathrm{p}}{z}\right)-\frac{1}{z} .
\end{array}
$$

On the other hand, it is unclear how one can make this argument to work rigorously. This would require a rather detailed investigation of the linear map $\mathcal{L}_{n-1}$ and recurrence (27), and this seems to be very technical.

\section{Appendix A}

A.1. Approach through $\mathrm{p}=0$. With a slight abuse of notation, we will use the expression $\frac{\partial^{s}}{\partial \mathrm{p}^{s}} G(0, z)$ to denote $\left.\frac{\partial^{s}}{\partial \mathrm{p}^{s}} G(\mathrm{p}, z)\right|_{\mathrm{p}=0}$ for $s \in \mathbb{N}_{0}$. Though the function $G(\mathrm{p}, z)$ is defined only for $\Re \mathrm{p} \geq 1, z \notin\left(\mathscr{I}_{\mathrm{p}}+1\right)$, assume that we are able to prove that it is analytic in $\mathrm{p}$ in a certain wider domain containing a disc $|\mathrm{p}|<\varpi$, $\varpi>0$. These are only formal calculations, but they unexpectedly yield series (7) (see Section 1), and numerical calculations do strongly confirm the validity of it.

Thus, substitution of $\mathrm{p}=0$ into (23) gives $G(0, z)=\frac{1}{2(1-z)}$. Partial differentiation of (23) with respect to $\mathrm{p}$, and consequent substitution of $\mathrm{p}=0$ gives

$$
\frac{1}{z^{2}} G(0,0)+2 \frac{\partial}{\partial \mathrm{p}} G(0, z+1)=G(0,0) \Rightarrow \frac{\partial}{\partial \mathrm{p}} G(0, z)=\frac{(z-1)^{2}-1}{4(z-1)^{2}} .
$$

In the same fashion, differentiating the second time, we obtain $\frac{\partial^{2}}{\partial \mathrm{p}^{2}} G(0, z)=$ $\frac{(z-1)^{4}-1}{2(z-1)^{3}}$. In general, differentiating (23) $n \geq 1$ times with respect to $\mathrm{p}$, using 
(24), and substituting $\mathrm{p}=0$, we obtain:

$$
2 \frac{\partial^{n}}{\partial \mathrm{p}^{n}} G(0, z+1)=\sum_{i+j=n-1} n\left(\begin{array}{c}
n-1 \\
j
\end{array}\right) \frac{\partial^{i} \partial^{j}}{\partial \mathrm{p}^{i} \partial z^{j}} G(0,0)\left(z^{j}-\frac{1}{z^{j+2}}\right) .
$$

Let

$$
\frac{1}{n !} \cdot \frac{\partial^{n}}{\partial \mathrm{p}^{n}} G(0, z)=\overline{\mathbf{Q}}_{n}(z)
$$

Then

$$
2 \overline{\mathbf{Q}}_{n}(z+1)=\sum_{j=0}^{n-1} \frac{1}{j !} \frac{\partial^{j}}{\partial z^{j}} \overline{\mathbf{Q}}_{n-j-1}(0)\left(z^{j}-\frac{1}{z^{j+2}}\right) .
$$

Consequently, we have a recurrent formula to compute rational functions $\overline{\mathbf{Q}}(z)$. Let $\mathbf{Q}_{n}(z)=\overline{\mathbf{Q}}_{n}(z+1)$. Thus,

$$
\mathbf{Q}_{n}(z)=\frac{(z+1)(z-1) \mathscr{D}_{n}(z)}{z^{n+1}}, \quad n \geq 1,
$$

where $\mathscr{D}_{n}$ are polynomials of degree $2 n-2$ with the reciprocity property $\mathscr{D}_{n}(z)=$ $z^{2 n-2} \mathscr{D}_{n}\left(\frac{1}{z}\right)$ (this is obvious from the recurrence relation which defines $\mathbf{Q}_{n}(z)$ ). Moreover, the coefficients of $\mathscr{D}_{n}$ are $\mathbb{Q}_{p}$ integers for any prime $p \neq 2$. These calculations yield the following formal result:

$$
G(\mathrm{p}, z) "=" \sum_{n=0}^{\infty} \mathrm{p}^{n} \cdot \mathbf{Q}_{n}(z-1)=\sum_{n=0}^{\infty} \mathrm{p}^{n} \frac{z(z-2) \mathscr{D}_{n}(z-1)}{(z-1)^{n+1}}
$$

This produces the "series" for the second and higher moments of the form

$$
m_{2}(\mathrm{p})=\mathrm{p}^{2} \cdot \sum_{n=0}^{\infty} \mathrm{p}^{n} \mathbf{Q}_{n}^{\prime}(-1)
$$

In particular, inspection of the table in Section 1 (where the initial values for $\mathbf{Q}_{n}^{\prime}(-1)$ are listed) shows that this series for $\mathrm{p}=1$ does not converge. However, the Borel sum is properly defined and it converges exactly to the value $m_{2}$. This gives empirical evidence for the validity of (7). The principles of Borel summation also suggest the mysterious fact that indeed $G(\mathrm{p}, z)$ analytically extends to the interval $\mathrm{p} \in[0,1]$.

Additionally, numerical calculations reveal the following fact: the sequence $\sqrt[n]{\left|\mathbf{Q}_{n}^{\prime}(-1)\right|}$ is monotonically increasing (apparently, tends to $\infty$ ), while $\frac{1}{n} \log \left|\mathbf{Q}_{n}^{\prime}(-1)\right|-\log n$ monotonically decreases (apparently, tends to $-\infty$ ). Thus,

$$
A^{n}<\left|\mathbf{Q}_{n}^{\prime}(-1)\right|<(c n)^{n},
$$

for $c=0.02372$ and $A=3.527, n \geq 150$. We do not have enough evidence to conjecture the real growth of this sequence. If $c=c(n) \rightarrow 0$, as $n \rightarrow \infty$, then the function

$$
\Lambda(t)=\sum_{n=0}^{\infty} \frac{\mathbf{Q}_{n}^{\prime}(-1)}{n !} t^{n}
$$

is entire, and in case $L=2$, result (7) is equivalent to the fact that

$$
\int_{0}^{\infty} \Lambda(t) e^{-t} \mathrm{~d} t=m_{2}
$$


A.2. Auxiliary lemmas. These lemmas are needed in Section 3. For $a, b \in \mathbb{N}$, $\mathrm{p} \in \mathbb{C},|\mathrm{p}-2| \leq 1$, define the rational functions

$$
W_{a}(\mathrm{p})=\frac{\mathrm{p}^{a}-1}{\mathrm{p}^{a+1}-\mathrm{p}^{a}}, \quad T_{a, b}(\mathrm{p})=W_{a}^{-1}(\mathrm{p}) W_{b}^{-1}(\mathrm{p}) \mathrm{p}^{-a}=\frac{(\mathrm{p}-1)^{2} \mathrm{p}^{b}}{\left(\mathrm{p}^{a}-1\right)\left(\mathrm{p}^{b}-1\right)} .
$$

Let us define constants

$$
\mu(a, b)=\sup _{\mathrm{p} \in \mathbb{C},|\mathrm{p}-2| \leq 1}\left|T_{a, b}(\mathrm{p})\right|-\Re\left(T_{a, b}(\mathrm{p})\right) .
$$

The following table provides some initial values for constants $\mu(a, b)$, computed numerically.

\begin{tabular}{|r|r|r|r|r|r|r|}
\hline$b \backslash a$ & 1 & 2 & 3 & 4 & 5 & 6 \\
\hline 1 & 0.25000000 & 0.01250000 & 0.00780868 & 0.03343231 & 0.05778002 & 0.07712952 \\
2 & 0.29846114 & 0.03125000 & 0.00159908 & 0.01212467 & 0.02539758 & 0.03645721 \\
3 & 0.35999295 & 0.05097235 & 0.00647895 & 0.00676996 & 0.01624300 & 0.02437494 \\
4 & 0.41433340 & 0.07007201 & 0.01316542 & 0.00500146 & 0.01287728 & 0.01963810 \\
5 & 0.45590757 & 0.08747624 & 0.02069451 & 0.00437252 & 0.01163446 & 0.01781467 \\
6 & 0.48390408 & 0.10255189 & 0.02845424 & 0.00812804 & 0.01125132 & 0.01728395 \\
7 & 0.49985799 & 0.11503743 & 0.03601828 & 0.01200557 & 0.01120308 & 0.01729854 \\
8 & 0.50642035 & 0.12494927 & 0.04309384 & 0.01611126 & 0.01125789 & 0.01748823 \\
9 & 0.50681483 & 0.13248892 & 0.04949922 & 0.02025219 & 0.01132055 & 0.01767914 \\
10 & 0.50452450 & 0.13796512 & 0.05514483 & 0.02427779 & 0.01136245 & 0.01780892 \\
11 & 0.50218322 & 0.14173414 & 0.06001269 & 0.02807992 & 0.01138335 & 0.01787452 \\
12 & 0.50070286 & 0.14415527 & 0.06413550 & 0.03158969 & 0.01139099 & 0.01789618 \\
13 & 0.49999979 & 0.14555794 & 0.06757752 & 0.03477145 & 0.01139235 & 0.01789583 \\
14 & 0.49977304 & 0.14622041 & 0.07041891 & 0.03761547 & 0.01139159 & 0.01788837 \\
15 & 0.49977361 & 0.14636154 & 0.07274403 & 0.04013040 & 0.01139057 & 0.01788111 \\
$\ldots$ & $\ldots$ & $\ldots$ & $\ldots$ & $\ldots$ & $\ldots$ & $\ldots$ \\
$\infty$ & 0.50000000 & 0.12500000 & 0.05479177 & 0.03097495 & 0.01138938 & 0.01787406 \\
\hline
\end{tabular}

Note that there exists $\lim _{b \rightarrow \infty} \mu(a, b)$, and $\mu(a, b) \rightarrow 0$ uniformly in $b$, as $a \rightarrow \infty$. Thus, the table above and some standard evaluations give the following

Lemma A.1. Let $a, b, c \in \mathbb{N}$. Then

$$
\mu(a, b)+\mu(b, c) \leq \mu(1,1)+\mu(1,9)<0.76 .
$$

Lemma A.2. There exists an absolute constant $c>0$ such that for all $\mathrm{p} \in \mathbb{C}$, $\Re \mathrm{p} \geq 1$, and all $a \in \mathbb{N}$, one has $\left|\frac{\mathrm{p}^{a}-1}{\mathrm{p}-1}\right|>c$.

Proof. Consider a contour, consisting of the segment $[1-i T, 1+i T]$, and a semicircle $1+T e^{i \phi},-\frac{\pi}{2} \leq \phi \leq \frac{\pi}{2}$. For sufficiently large $T, \frac{\mathrm{p}^{a}-1}{\mathrm{p}-1}$ will be large on the semicircle. Moreover, this function never vanishes inside or on the contour. Thus, from the maximum-minimum principle, its minimal absolute value is obtained on the segment $[1-i T, 1+i T]$. Thus, let $\mathrm{p}=\frac{1}{\cos \psi} e^{i \psi},-\frac{\pi}{2}<\psi<\frac{\pi}{2}$. Without loss of generality, let $\psi \geq 0$. Consider the case $\frac{\pi}{2 a} \leq \psi<\frac{\pi}{2}$. Then

$$
\left|\frac{\mathbf{p}^{a}-1}{\mathbf{p}-1}\right|^{2}=\frac{\frac{1}{\cos ^{2 a} \psi}-\frac{2 \cos a \psi}{\cos ^{a} \psi}+1}{\frac{1}{\cos ^{2} \psi}-1} \geq \frac{\frac{1}{\cos ^{2 a} \psi}-\frac{2}{\cos ^{a} \psi}+1}{\frac{1}{\cos ^{2} \psi}-1}=\frac{\left(\rho^{a}-1\right)^{2}}{\rho^{2}-1}:=Y(\rho), \quad \rho=\frac{1}{\cos \psi} .
$$


The function $Y(\rho)$ is an increasing function in $\rho$ for $\rho \geq 1$. It is obvious that we may consider a case of $a$ sufficiently large. Thus,

$$
\begin{aligned}
\left|\frac{\mathrm{p}^{a}-1}{\mathrm{p}-1}\right|^{2} & \geq Y\left(\frac{1}{\cos \frac{\pi}{2 a}}\right)=\frac{\left(\frac{1}{\cos ^{a} \frac{\pi}{2 a}}-1\right)^{2}}{\tan ^{2} \frac{\pi}{2 a}} \\
& =\frac{\left(\left(1+\frac{\pi^{2}}{8 a^{2}}+\frac{\mathcal{O}(1)}{a^{3}}\right)^{a}-1\right)^{2}}{\frac{\pi^{2}}{4 a^{2}}+\frac{\mathcal{O}(1)}{a^{3}}}=\frac{\frac{\pi^{4}}{64 a^{2}}+\frac{\mathcal{O}(1)}{a^{3}}}{\frac{\pi^{2}}{4 a^{2}}+\frac{\mathcal{O}(1)}{a^{3}}}=\frac{\pi^{2}}{16}+\frac{\mathcal{O}(1)}{a} .
\end{aligned}
$$

Now let $0 \leq \psi<\frac{\pi}{2 a}$. First, consider a function $\frac{1}{y} \log \cos (y \psi):=V(y)$. It is a decreasing function for $0<y<\frac{\pi}{2 \psi}$. Indeed, this is equivalent to the inequality

$$
-\tan x \cdot x-\log \cos x<0, \text { for } 0<x<\frac{\pi}{2} .
$$

The function on the left is itself a decreasing function, with maximum value attained at $x=0$. Thus, $V(1) \geq V(a)$, which means $\cos a \psi \leq \cos ^{a} \psi$, and this gives

$$
\left|\frac{\mathrm{p}^{a}-1}{\mathrm{p}-1}\right|^{2} \geq \frac{\frac{1}{\cos ^{2 a} \psi}-1}{\frac{1}{\cos ^{2} \psi}-1} \geq 1 .
$$

Therefore, Lemma A.2 implies that the function $\mathrm{p}^{-1} W_{a}^{-1}(\mathrm{p})$ is uniformly bounded:

$$
\sup _{a \in \mathbb{N},|\mathrm{p}-2| \leq 1}\left|\mathrm{p}^{-1} W_{a}^{-1}(\mathrm{p})\right|=c<+\infty .
$$

This shows the validity of the following lemma (apart from a numerical bound, which is the outcome of computer calculations).

Lemma A.3. One has

$$
\sup _{|\mathrm{p}-2| \leq 1, a \in \mathbb{N},|z-1| \leq 1}\left|\mathrm{p}^{-1} W_{a}^{-1}(\mathrm{p}) z-1\right|<1.29 .
$$

A.3. Numerical values for the moments. Unfortunately, Corollary 1.4 is not very useful in finding exact decimal digits of $m_{2}$. In fact, the vector $\left(m_{1}, m_{2}, m_{3}, \ldots\right)$ is the solution of an (infinite) system of linear equations, which encodes the functional equation (6) (see [1], Proposition 6). Namely, if we denote $c_{L}=\sum_{n=1}^{\infty} \frac{1}{2^{n} n^{L}}=$ $\operatorname{Li}_{L}\left(\frac{1}{2}\right)$, we have a linear system for $m_{s}$ which describes the coefficients $m_{s}$ uniquely:

$$
m_{s}=\sum_{L=0}^{\infty}(-1)^{L} c_{L+s}\left(\begin{array}{c}
L+s-1 \\
s-1
\end{array}\right) m_{L}, \quad s \geq 1 .
$$

Note that this system is not homogeneous $\left(m_{0}=1\right)$. We truncate this matrix at sufficiently high order to obtain float values. The accuracy of this calculation can be checked on the test value $m_{1}=0.5$. This approach yields (for the matrix of order 325):

$$
\begin{aligned}
& m_{2}=0.2909264764293087363806977627391202900804371021955943665492_{+}, \\
& m_{3}=0.1863897146439631045710466441086804351206556532933915498238_{+}, \\
& m_{4}=0.1269922584074431352028922278802116388411851457617257181016_{+},
\end{aligned}
$$


with all 58 digits exact (note that $3 m_{2}-2 m_{3}=0.5$ ). In fact, the truncation of this matrix at an order 325 gives rather accurate values for $m_{L}$ for $1 \leq L \leq 125$, well in correspondence with an asymptotic formula [3]

$$
m_{L}=\sqrt[4]{4 \pi^{2} \log 2} \cdot c_{0} \cdot L^{1 / 4} \mathrm{C}^{\sqrt{L}}+\mathcal{O}\left(\mathrm{C}^{\sqrt{L}} L^{-1 / 4}\right)
$$

where $c_{0}=\int_{0}^{1} 2^{x}(1-F(x)) \mathrm{d} x=1.030199563382_{+}, \mathrm{C}=e^{-2} \sqrt{\log 2}$. The numerical values for higher moments so obtained tend to deviate from this expression rather quickly.

Kinney [16] proved that the Hausdorff dimension of growth points of ? $(x)$ is equal to

$$
\alpha=\frac{1}{2}\left(\int_{0}^{1} \log _{2}(1+x) \mathrm{d} ?(x)\right)^{-1} .
$$

Lagarias [19] gives the following estimates: $0.8746<\alpha<0.8749$. Tichy and Uitz 33. calculated $\alpha \approx 0.875$. Paradís et al. [26] give the value $\alpha \approx 0.874832$. We have (note that $?(1-x)+?(x)=1)$ :

$$
\begin{array}{r}
A:=\int_{0}^{1} \log (1+x) \mathrm{d} ?(x)=\int_{0}^{1} \log \left(1-\frac{1-x}{2}\right) \mathrm{d} ?(x)+\int_{0}^{1} \log 2 \mathrm{~d} ?(x) \\
\quad=-\sum_{L=1}^{\infty} \frac{1}{L \cdot 2^{L}} \int_{0}^{1}(1-x)^{L} \mathrm{~d} ?(x)+\log 2=-\sum_{L=1}^{\infty} \frac{m_{L}}{L \cdot 2^{L}}+\log 2 .
\end{array}
$$

Thus, we are able to present a much more precise result:

$$
\alpha=\frac{\log 2}{2 A}=0.874716305108211142215152904219159757_{+}
$$

with all 36 digits exact. The author of this paper has contacted the authors of 26] inquiring about the error bound for the numerical value of $\alpha$ they obtained. It appears that for this purpose 10 generations of (2) were used. The authors of [26] were very kind in agreeing to perform the same calculations with more generations. Thus, if one uses 18 generations, this gives $0.874716<\alpha<0.874719$.

Additionally, the constant $c_{0}$ in (28) is given by

$$
c_{0}=\int_{0}^{1} 2^{x}(1-F(x)) \mathrm{d} x=\frac{\mathfrak{m}(\log 2)}{2 \log 2}=\frac{1}{2} \sum_{L=0}^{\infty} \frac{m_{L}}{L !}(\log 2)^{L-1} .
$$

This series is fast convergent, and we obtain

$$
c_{0}=1.03019956338269462315600411256447867669415885918240_{+}
$$

A.4. Rational functions $\mathbf{H}_{n}(z)$. The following is MAPLE code for computing rational functions:

$\mathbf{H}_{n}(z)=\mathrm{h}[\mathrm{n}]$ and coefficients

$\mathbf{H}_{n}^{\prime}(0)=$ alpha $[\mathrm{n}]$ for $0 \leq n \leq 50$.

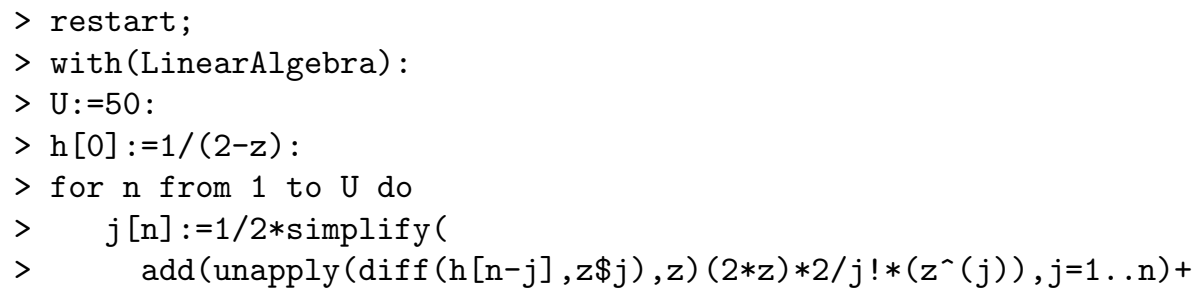




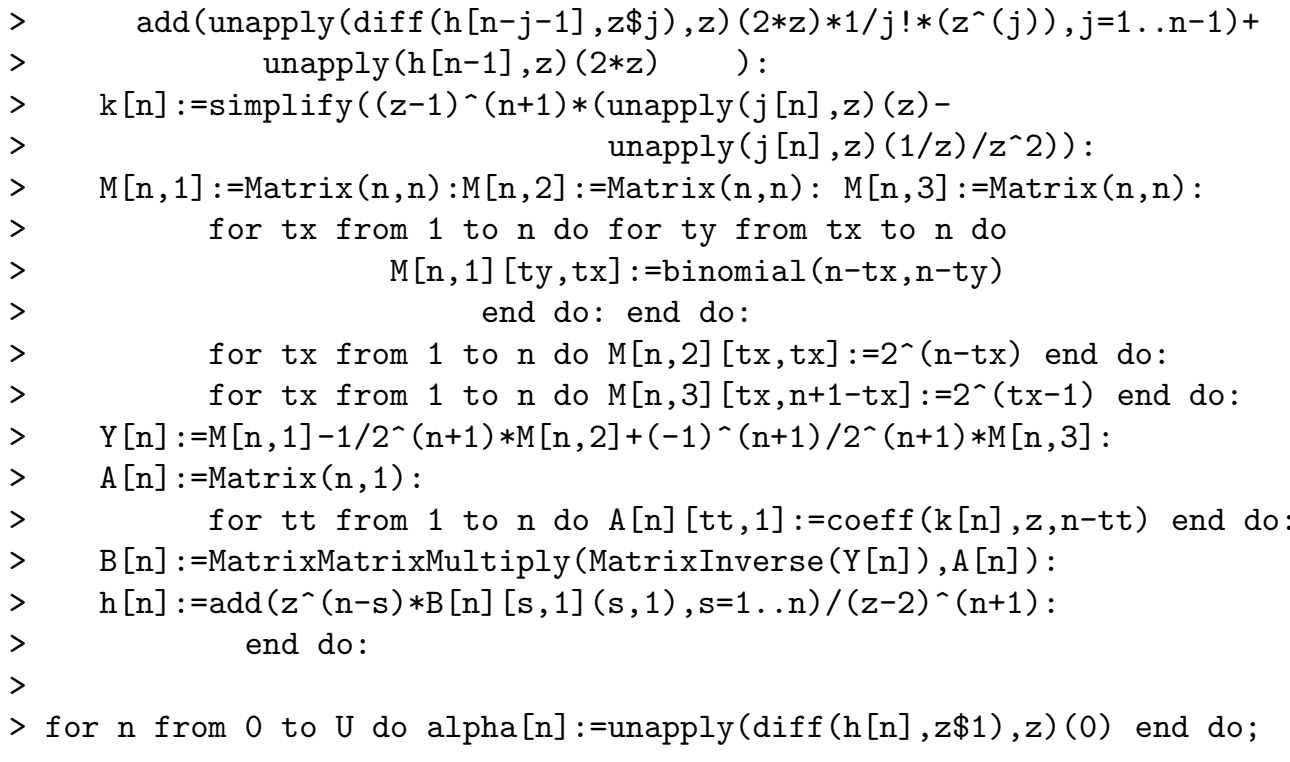

It causes no complications to compute $\mathrm{h}[\mathrm{n}]$ on a standard home computer for $0 \leq n \leq 60$, though the computations heavily increase in difficulty for $n>60$.

A.5. Rational functions $\mathbf{Q}_{n}(z)$. This program computes $\mathbf{Q}_{n}(z)=\mathrm{q}[\mathrm{n}]$ and the values

$\mathbf{Q}_{n}^{\prime}(-1)=\operatorname{beta}[\mathrm{n}]$ for $0 \leq n \leq 50$.

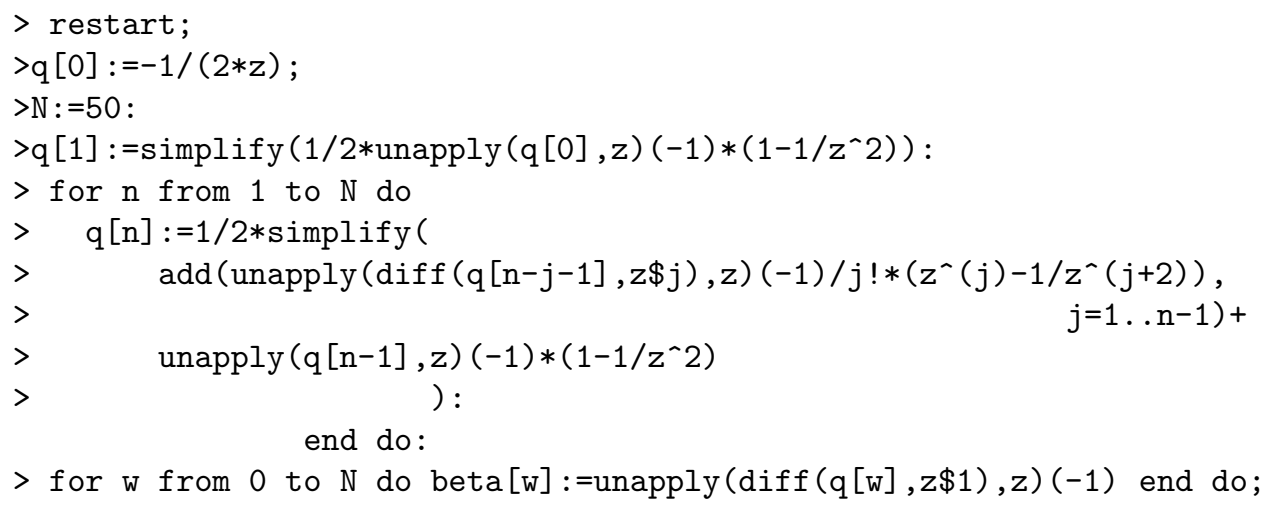




\section{ACKNOWLEDGEMENTS}

The author sincerely thanks Jörn Steuding, whose seemingly elementary problem, proposed at the problem session at the Palanga conference in 2006, turned out to be a very rich and generous one. Also, the author thanks several colleagues for showing interest in these results, after preprints became available in arXiv, especially to Steven Finch, Jeffrey Lagarias and Stefano Isola.

Part of the work on this paper was done at the Max Planck Institute for Mathematics, and the author acknowledges support from the research grant of the institute.

\section{REFERENCES}

[1] G. Alkauskas, The moments of Minkowski question mark function: the dyadic period function (submitted); arXiv:0801.0051.

[2] G. Alkauskas, Generating and zeta functions, structure, spectral and analytic properties of the moments of the Minkowski question mark function, Involve (to appear); arXiv:0801.0056.

[3] G. Alkauskas, An asymptotic formula for the moments of Minkowski question mark function in the interval [0,1], Lith. Math. J. 48 (4) 2008, 357-367.

[4] A. F. Beardon, Iteration of rational functions, Complex analytic dynamical systems, Springer-Verlag, 1991. MR 1128089 (92j:30026)

[5] C. Bonanno, S. Graffi, S. Isola, Spectral analysis of transfer operators associated to Farey fractions, Atti Accad. Naz. Lincei Cl. Sci. Fis. Mat. Natur. Rend. Lincei (9) Mat. Appl. 19 (1) (2008), 1-23. MR2383559

[6] C. Bonanno, S. Isola, Orderings of the rationals and dynamical systems, Colloq. Math. (to appear); arXiv:0805.2178.

[7] T. Bousch, Connexité locale et par chemins hölderiens pour les systèmes itérés de fonctions; Available at http://topo.math.u-psud.fr/ bousch (1993) (unpublished).

[8] N. Calkin, H. Wilf, Recounting the rationals, Amer. Math. Monthly 107 (4) (2000), 360363. MR 1763062 (2001d:11024)

[9] A. Denjoy, Sur une fonction réelle de Minkowski, J. Math. Pures Appl. 17 (1938), 105-151.

[10] A. Dushistova, N.G. Moshchevitin, On the derivative of the Minkowski question mark function? $(x)$; arXiv:0706.2219

[11] M.D. Esposti, S. Isola, A. Knauf, Generalized Farey trees, transfer operators and phase transitions, Comm. Math. Phys. 275 (2) (2007), 297-329. MR2335777 (2008i:37043)

[12] R. Girgensohn, Constructing singular functions via Farey fractions, J. Math. Anal. Appl. 203 (1) (1996), 127-141. MR1412484 (97f:26006)

[13] P. J. Grabner, P. Kirschenhofer, R. Tichy, Combinatorial and arithmetical properties of linear numeration systems, Combinatorica 22 (2) (2002), 245-267. MR.1909085 (2003f:11113)

[14] M. Kesseböhmer, B.O. Stratmann, Fractal analysis for sets of non-differentiability of Minkowski's question mark function; J. Number Theory 128 (9) (2008), 2663-2686. MR2444218

[15] A. YA. Khinchin, Continued fractions, The University of Chicago Press, 1964. MR 0161833 $(28: 5037)$

[16] J.R. Kinney, Note on a singular function of Minkowski, Proc. Amer. Math. Soc. 11 (5) (1960), 788-794. MR0130330 (24:A194)

[17] S. Klavžar, U. Milutinović, C. Petr, Stern Polynomials. Adv. in Appl. Math. 39 (1) (2007), 86-95. MR.2319565 (2008c:11033)

[18] J.C. Lagarias, The Farey shift and the Minkowski ?-function, (preprint).

[19] J. C. Lagarias, Number theory and dynamical systems, The unreasonable effectiveness of number theory (Orono, ME, 1991), Proc. Sympos. Appl. Math., 46, Amer. Math. Soc. (1992), 35-72. MR1195841 (93m:11143)

[20] J.C. Lagarias, C.P. Tresser, A walk along the branches of the extended Farey tree, IBM J. Res. Develop. 39 (3) May (1995), 788-794. MR2361372

[21] M. LAmBerger, On a family of singular measures related to Minkowski's ?(x) function, Indag. Math. (N.S.), 17 (1) (2006), 45-63. MR2337164(2008g:60116) 
[22] J.B. LEwIS, Spaces of holomorphic functions equivalent to the even Maass cusp forms, Invent. Math. 127 (2) (1997), 271-306. MR1427619(98f:11036)

[23] J.B. Lewis, D. Zagier, Period functions for Maass wave forms. I, Ann. of Math. (2) 153 (1) (2001), 191-258. MR1826413 (2003d:11068)

[24] H. Окамото, M. Wunsch, A geometric construction of continuous, strictly increasing singular functions, Proc. Japan Acad. Ser. A Math. Sci. 83 (7) (2007), 114-118. MR2361422 (2008k:26008)

[25] G. Panti, Multidimensional continued fractions and a Minkowski function, Monatsh. Math. 154 (3) (2008), 247-264. MR2413304

[26] J. Paradís, P. Viader, L. Bibiloni, The derivative of Minkowski's ?(x) function. J. Math. Anal. Appl. 253 (1) (2001), 107-125. MR.1804596 (2002c:11092)

[27] J. Paradís, P. Viader, L. Bibiloni, A new light on Minkowski's ? $(x)$ function, J. Number Theory 73 (2) (1998), 212-227. MR 1658027(2000a:11104)

[28] G. Ramharter, On Minkowski's singular function, Proc. Amer. Math. Soc., 99 (3) (1987), 596-597. MR875407 (88c:11013)

[29] S. ReEse, Some Fourier-Stieltjes coefficients revisited. Proc. Amer. Math. Soc. 105 (2) (1989), 384-386. MR938913 (89i:42020)

[30] F. Ryde, On the relation between two Minkowski functions, J. Number Theory, 17 (1) (1983), 47-51. MR712967 (85b:11008)

[31] R. Salem, On some singular monotonic functions which are strictly increasing, Trans. Amer. Math. Soc. 53 (3) (1943), 427-439. MR0007929(4:217b)

[32] B. Solomyak, On the 'Mandelbrot set' for pairs of linear maps: Asymptotic self-similarity, Nonlinearity 18 (5) (2005), 1927-1943. MR2164725 (2006d:37086)

[33] R. F. Тiснy, J. Uitz, An extension of Minkowski's singular function, Appl. Math. Lett. 8 (5) (1995), 39-46. MR 1356295 (96i:26005)

[34] H. S. WALL, Analytic theory of continued fractions, D. Van Nostrand Company, Inc., New York, N. Y., 1948. MR0025596(10:32d)

[35] G. N. Watson, A treatise on the theory of Bessel functions, 2nd ed. Cambridge University Press, 1996. MR.1349110 (96i:33010)

[36] An exhaustive bibliography on the Minkowski question mark function, Available at http://www.maths.nottingham.ac.uk/personal/pmxga2/minkowski.htm

The Department of Mathematics and Informatics, Vilnius University, Naugarduko 24, Vilnius, Lithuania and Max-Planck-Institut für Mathematik, Vivatsgasse 7, 53111 Bonn, Germany

E-mail address: giedrius.alkauskas@gmail.com 